\title{
Compound real Wishart and $q$-Wishart matrices
}

\author{
Włodzimierz Bryc ${ }^{\mathrm{a}}$ \\ a Department of Mathematical Sciences, University of Cincinnati, 2855 Campus Way, PO Box 210025, Cincinnati, OH \\ 45221-0025, USA. Email: Wlodzimierz.Bryc@UC.edu
}

\begin{abstract}
We introduce a family of matrices with non-commutative entries that generalize the classical real Wishart matrices. With the help of the Brauer product, we derive a non-asymptotic expression for the moments of traces of monomials in such matrices; the expression is quite similar to the formula derived in 9, Theorem 2.1] for independent complex Wishart matrices. We then analyze the fluctuations about the Marchenko-Pastur law. We show that after centering by the mean, traces of real symmetric polynomials in $q$-Wishart matrices converge in distribution, and we identify the asymptotic law as the normal law when $q=1$, and as the semicircle law when $q=0$.
\end{abstract}

KEY WORDS Compound Wishart matrices, Central Limit Theorem, matrices with noncommutative entries, $q$-Gaussian random variables, fluctuations about the Marchenko-Pastur law

Received October 5, 2007; Revised March 13, 2008

\section{Introduction}

The real Wishart distribution was introduced by Wishart [46. As evidenced by the vast literature, the Wishart law is of primary importance to statistics, see e.g. [1, 35. The compound Wishart distribution was introduced by Speicher 41. We will consider a slightly more general class of laws on random matrices which was introduced in Ref. 20, and also its non-commutative generalization.

Definition 1.1. Let $\Sigma$ be an $N \times N$ real positive-definite matrix (i.e. $x^{*} \Sigma x>0$ for all non-zero $x \in \mathbb{R}^{N}$ ). Let $B$ be an $M \times M$ real matrix. We will say that a random matrix $\mathbf{W}$ is Wishart with shape parameter $B$ and scale parameter $\Sigma$, if

$$
\mathbf{W}=A^{\prime} \mathbf{X}^{\prime} B \mathbf{X} A
$$

where $\mathbf{X}$ is an $M \times N$ matrix of i.i.d. $N(0,1)$ random variables, and $A$ is a symmetric root, $A^{\prime} A=A^{2}=\Sigma$. (Here and throughout the paper, $A^{\prime}$ denotes the transpose of $A$.) We write $\mathbf{W} \in \mathcal{W}(\Sigma, B)$.

When $B$ is positive-definite, Ref. [10] interprets $\mathbf{W}$ as a sample covariance under correlated sampling. Similar interpretation for the complex case appears in Ref. 11. Somewhat more generally, one could consider all matrices of the form (1.1) with non-symmetric $A$; the moments of such matrices would then depend on $\Sigma^{+}=A^{\prime} A$ and $\Sigma^{-}=A A^{\prime}$ as well as on $B, B^{\prime}$.

The usual real Wishart matrices correspond to the choice $B=I_{M}$, so the notation $W(\Sigma, M)$ in [1, Section 7.2] means $\mathcal{W}\left(\Sigma, I_{M}\right)$ in our notation. Ref. [17] uses a slightly different parametrization $\sigma=2 \Sigma, p=M / 2$, which should be kept in mind when comparing the formulas. Compound Wishart matrices are the elements of $\mathcal{W}\left(I_{N}, B\right)$, see [41, Section 4.4] or [21, page 169].

Our main result about real Wishart matrices, Theorem 2.9 gives a non-asymptotic expression for the moments of traces of monomials in several independent real Wishart matrices. This formula is similar to the expression derived in 9, Theorem 2.1] for independent complex Wishart matrices. The statement requires additional notation on Brauer products which will be introduced in Section 2 Our main application of this formula deals with the fluctuations about the Marchenko-Pastur law. The latter problem fits naturally into a more general non-commutative setting, see Theorem 1.7

We now introduce matrices which arise from matrices with noncommutative $q$-Gaussian entries by a formula similar to (1.1). The noncommutative $q$-Gaussian random variables were introduced on the algebraic level by Frisch and Bourret [14; additional physical applications are in [38, 44. The Fock-space construction is due to Bożejko and Speicher [7] an excellent exposition of this topic appears in Ref. 6].

Fix a parameter $-1 \leq q \leq 1$. Let $\mathcal{A}$ be a noncommutative probability space, i.e., a unital complex $*$-algebra with a tracial state $\tau: \mathcal{A} \rightarrow \mathbb{C}$; see, e. g., [21, Sect. 1.2] or [45] for more details and examples. 
Definition 1.2 ([14, Theorem 1]). $\mathbf{X}_{1}, \mathbf{X}_{2}, \cdots \in \mathcal{A}$ are $q$-Gaussian, if $\mathbf{X}_{j}=\mathbf{X}_{j}^{*}$, and for any finite collection of $\mathbf{Y}_{1}, \ldots, \mathbf{Y}_{n} \in\left\{\mathbf{X}_{1}, \mathbf{X}_{2}, \ldots\right\}$,

$$
\tau\left(\mathbf{Y}_{1} \ldots \mathbf{Y}_{n}\right)=\sum_{\mathcal{V}} q^{\operatorname{cr}(\mathcal{V})} \prod_{\{i, j\} \in \mathcal{V}} \tau\left(\mathbf{Y}_{i} \mathbf{Y}_{j}\right)
$$

The sum is over all pair partitions $\mathcal{V}$ of $\{1, \ldots, n=2 k\}$ and to be interpreted as 0 when $n$ is odd; $\operatorname{cr}(\mathcal{V}):=$ $\#\left\{\left(i_{1}, i_{2}\right):\left\{i_{1}, j_{1}\right\},\left\{i_{2}, j_{2}\right\} \in \mathcal{V}, i_{1}<i_{2}<j_{1}<j_{2}\right\}$ is the number of crossings of a pair partition $\mathcal{V}$.

Consider now the $*$-probability space $\mathcal{M}_{N \times N}(\mathcal{A})$ of all $N \times N$ matrices with elements from $\mathcal{A}$, with matrix multiplication as the product operation, with state

$$
\tau \circ \operatorname{tr}_{N}(\mathbf{X}):=\frac{1}{N} \sum_{i=1}^{N} \tau\left([\mathbf{X}]_{i, i}\right),
$$

and with the natural definition of the star operation as conjugation of elements in $\mathcal{A}$, and transposition.

For $N \geq 1$, a positive definite real $N \times N$ matrix $\Sigma$ and an $M \times M$ positive definite real matrix $B$, let $\mathbf{X}_{i, j}$ be $q$-Gaussian noncommutative random variables such that for $1 \leq i, k \leq M, 1 \leq j, m \leq N$,

$$
\tau\left(\mathbf{X}_{i, j} \mathbf{X}_{k, m}\right)=[B]_{i, k}[\Sigma]_{j, m} .
$$

Definition 1.3. We will say that $\mathbf{W} \in \mathcal{M}_{N \times N}(\mathcal{A})$ is a $q$-Wishart random variable with shape parameter $B$ and scale parameter $\Sigma$ if

$$
\mathbf{W}=\mathbf{X}^{*} \mathbf{X}
$$

where $\mathbf{X}=\left[\mathbf{X}_{i, j}\right]$ is the $M \times N$ matrix with q-Gaussian entries that satisfy (1.2). We will write $\mathbf{W} \in \mathcal{W}_{q}(\Sigma, B)$.

(Matrices with noncommutative entries have been studied in [36, 39, 40, 43]. According to [30, Definition 1.2.2] when $\Sigma=I_{N}, B=I_{M}, \mathbf{X}_{i, j}$ form a $q$-circular system.)

We will also need a substitute for independence.

Definition 1.4. We will say that $\mathbf{W}_{1}, \mathbf{W}_{2}, \ldots, \mathbf{W}_{s} \in \mathcal{W}_{q}(\Sigma, B)$ are q-orthogonal if the entries of the corresponding matrices (1.3) : $\left\{\mathbf{X}_{i, j}^{(1)}\right\}$ for $\mathbf{W}_{1},\left\{\mathbf{X}_{i, j}^{(2)}\right\}$ for $\mathbf{W}_{2}, \ldots$, are pairwise uncorrelated, $\tau\left(\mathbf{X}_{i, j}^{(k)} \mathbf{X}_{i^{\prime}, j^{\prime}}^{\left(k^{\prime}\right)}=\right.$ $[B]_{i, i^{\prime}}[\Sigma]_{j, j^{\prime}} \delta_{k, k^{\prime}}$.

Remark 1.5. When $q=1$, all $q$-Gaussian random variables commute and their joint moments are the moments of the multivariate normal law of matching covariance. In particular, for every finite $q$-Gaussian family $\mathbf{X}_{1}, \ldots, \mathbf{X}_{m}$, there exists an $m \times m$ real matrix $A$ and independent classical standard normal random variables $Z_{1}, \ldots, Z_{m}$ such that with $Y_{j}=\sum_{k}[A]_{j k} Z_{k}$,

$$
\tau\left(\mathbf{X}_{1}^{k_{1}} \ldots \mathbf{X}_{m}^{k_{m}}\right)=\mathbb{E}\left(Y_{1}^{k_{1}} \ldots Y_{m}^{k_{m}}\right) \text { for all } k_{1}, \ldots, k_{m} \geq 0 .
$$

Thus the joint moments of $q$-orthogonal $\mathbf{W}_{1}, \mathbf{W}_{2}, \ldots, \mathbf{W}_{s} \in \mathcal{W}_{q}(\Sigma, B)$ coincide with the respective joint moments of independent real Wishart matrices. Under appropriate assumptions, such matrices are asymptotically free and their limit laws are compound Marchenko Pastur laws. The conclusion of Theorem 1.7 in this case means that for real Wishart matrices the fluctuations around the Marchenko-Pastur law are asymptotically normal. (This fact is known for complex Wishart matrices, see [33.)

Remark 1.6. When $q=0$, for every finite $q$-Gaussian family $\mathbf{X}_{1}, \ldots, \mathbf{X}_{m}$, there exists an $m \times m$ real matrix $A$ and free semicircular elements $\mathbf{Z}_{1}, \ldots, \mathbf{Z}_{m}$ such that with $\mathbf{Y}_{j}=\sum_{k}[A]_{j k} \mathbf{Z}_{k}$,

$$
\tau\left(\mathbf{X}_{j_{1}} \ldots \mathbf{X}_{j_{n}}\right)=\tau\left(\mathbf{Y}_{j_{1}} \ldots \mathbf{Y}_{j_{n}}\right) \text { for all } 1 \leq j_{1}, \ldots, j_{n} \leq m \text {. }
$$

Thus, for example, the traces of powers of $q$-orthogonal $\mathbf{W}_{1}, \mathbf{W}_{2}, \ldots, \mathbf{W}_{s} \in \mathcal{W}_{q}(\Sigma, B)$ are free in $(\mathcal{A}, \tau)$. In Proposition 2.19 we show that $\mathbf{W} \in \mathcal{W}_{q}\left(\frac{1}{N} I_{N}, B\right)$ as an element of $\left(\mathcal{M}_{N \times N}(\mathcal{A}), \tau \circ \operatorname{tr}_{N}\right)$ has a compound Marchenko-Pastur law already for finite $N$, so on average the fluctuations around the limit are zero. Theorem 1.7 shows that the un-averaged fluctuations around the Marchenko-Pastur law have a non-trivial semicircular limit.

Asymptotic normality for traces of polynomials in several independent complex Wishart matrices is established in Ref. [33. This case served as a departure point for a number of papers on second order freeness, see e.g. [12] and the references therein. For an extension to more general covariances see also [9, Theorem 1]. Asymptotic normality for one real Wishart matrix is established in [2, Theorem 2], 23, Theorem 4.1]. Asymptotic normality of self-adjoint polynomials in noncommutative entries in a different setting appears in [24, Theorem 1]. It turns out that in our noncommutative setting, asymptotic limit law always exists, but it depends on the polynomial in a nontrivial way. 
Theorem 1.7. Suppose $\mathbf{W}_{1}^{(N)}, \mathbf{W}_{2}^{(N)}, \ldots, \mathbf{W}_{s}^{(N)} \in \mathcal{W}_{q}\left(\frac{1}{N} I_{N}, I_{M}\right)$ are q-orthogonal and $M=M(N)$ is such that $M / N \rightarrow \lambda \in[0, \infty)$ and $N \rightarrow \infty$. If $Q()$ is a fixed non-commutative polynomial in s variables, and

$$
X_{N}:=\operatorname{tr}\left(Q\left(\mathbf{W}_{1}^{(N)}, \ldots, \mathbf{W}_{s}^{(N)}\right)\right)-\tau\left(\operatorname{tr}\left(Q\left(\mathbf{W}_{1}^{(N)}, \ldots, \mathbf{W}_{s}^{(N)}\right)\right)\right)
$$

then $X_{N}$ converges in moments as $N \rightarrow \infty$. Furthermore, if $Q$ is a real symmetric polynomial so that $X_{N}$ is self-adjoint, then $X_{N}$ converges in distribution; if in addition $q=1$ then $X_{N}$ has asymptotically normal law; if $q=0$ then $X_{N}$ has asymptotically semicircle law.

Theorem 1.7 raises an interesting question about how to identify or describe the limiting law for all $q \in[-1,1]$. Our proof relies on a general property of $q$-Wishart matrices, see Theorem 4.1 and freeness or independence when $q=0$ or $q=1$; this technique is not helpful in general.

Computer-assisted calculations based on exact formulas from Theorem 2.18 indicate that the limit law for general $q$ depends on the polynomial in a complicated way, see Example 4.8. Similar situation was noted for Wigner matrices in noncommutative entries in an unpublished manuscript 31.

For one matrix case, one may be able to find polynomials with limits that can still be described in simple terms. One such polynomial seems to be $\operatorname{tr}\left(\mathbf{W}^{(N)}\right)$, see Example 4.6] another suggestive computer-assisted calculation is in Example 4.7 .

We now list two corollaries. We begin with traces of polynomials of a single $q$-Wishart matrix.

Corollary 1.8. If $Q$ is a real polynomial, $\mathbf{W} \in \mathcal{W}_{q}\left(\frac{1}{N} I_{N}, I_{M}\right), M / N \rightarrow \lambda \in[0, \infty)$, then

$$
X_{N}:=\operatorname{tr}(Q(\mathbf{W}))-\tau(\operatorname{tr}(Q(\mathbf{W})))
$$

converges in distribution as $N \rightarrow \infty$. Furthermore, if $q=1$ then $X_{N}$ has asymptotically normal law; if $q=0$ then $X_{N}$ has asymptotically semicircle law.

For $q=1$, the traces commute, and their joint moments coincide with the moments of traces of the corresponding real Wishart matrices (1.1); taking $Q\left(\mathbf{W}_{1}, \ldots, \mathbf{W}_{s}\right)=\sum_{j=1}^{k} a_{j} \prod_{r=1}^{j} \mathbf{W}_{t(r)}$, by the Cramer-Wald device ([4, Theorem 29.4]) we deduce the following multimatrix generalization of [2, Theorem 2], 23, Theorem 4.1] which shows that [33, Corollary 9.4] holds also for real Wishart matrices.

Corollary 1.9. Fix $s \geq 1$ and $t: \mathbb{N} \rightarrow\{1, \ldots, s\}$. Suppose $\mathbf{W}_{1}, \ldots, \mathbf{W}_{s} \in \mathcal{W}\left(\frac{1}{N} I_{N}, I_{M}\right)$ are independent. For a fixed $k \geq 1$ that does not depend on $N$, and for $1 \leq j \leq k$ consider real random variables

$$
X_{j}^{(N)}=\operatorname{tr}\left(\prod_{r=1}^{j} \mathbf{W}_{t(r)}\right)-\mathbb{E}\left(\operatorname{tr}\left(\prod_{r=1}^{j} \mathbf{W}_{t(r)}\right)\right) .
$$

Then as $N \rightarrow \infty, M / N \rightarrow \lambda \in[0, \infty)$, the sequence of $k$-dimensional random variables

$$
\left(X_{j}^{(N)}\right)_{1 \leq j \leq k}
$$

converges in distribution to the multivariate normal law.

The paper is organized as follows. In Section 2 we introduce notation, state our results on mixed moments of traces of monomials in independent Wishart matrices and on mixed moments of traces of monomials in $q$-orthogonal $q$-Wishart matrices, and we deduce some of the related expressions available in the literature. Section 3 contains proofs of the formulas for moments. Section 4 contains some auxiliary results, and the proof of Theorem 1.7 .

\section{Moments of real Wishart and $q$-Wishart matrices}

Explicit formulas for moments of polynomials in one real Wishart matrix appear in [17, 18, 20, 28. The formulas are more complicated than the formulas for the complex case [16, 20, 33, and often involve sophisticated tools, like wreath products, Jack polynomials, and properties of hyperoctahedral group.

One of the main results of this paper is the non-asymptotic expression for moments of traces of monomials in several independent real Wishart matrices, and its noncommutative $q$-generalization. In Section 4 we use the moment formula to prove Theorem [1.7 but since the formula is of independent interest we consider a more general case than what we need for the proof. Our approach follows closely [9, Theorem 2]: it relies on Wick formula, combinatorics from [33] and the Brauer product [8] of pair partitions. The connection of the Brauer 
algebra to integration over the orthogonal group has already been noted in 13 who seem not to use the Brauer product explicitly. With the use of the Brauer product, our formula closely resembles the formula for the complex case.

Following the referee's comment we remark that we use Brauer algebra as a convenient notational replacement for the permutation group, but since we multiply only by elements from $\mathcal{F}_{n}^{+}$, our use of the Brauer structure is in fact the action of $S_{n}$ on $2 n$-pair partitions, as in [17. In particular, the constant of the Brauer algebra does not enter our formulas.

After the first version of this paper was written, we learned from J. Mingo about his work 32 with Emily Redelmeier who has found a very elegant diagrammatic interpretation of the right hand side of equation (2.9).

\subsection{Pair partitions, permutations, Brauer product}

Consider matrix variables $\mathbf{x}_{1}, \mathbf{x}_{2}, \ldots, \mathbf{x}_{s}$ which we interpret as variables of "colors" $1, \ldots, s$. We are interested in monomials $p_{\gamma, t}\left(\mathbf{x}_{1}, \mathbf{x}_{2}, \ldots, \mathbf{x}_{s}\right)$ of degree $n$ which we parameterize by perfect matchings (pair partitions) $\gamma$ of $\{ \pm 1, \pm 2, \ldots, \pm n\}$, and by functions $t:\{1, \ldots, n\} \rightarrow\{1, \ldots, s\}$, which we interpret as assigning "colors" to the integers. To introduce these polynomials in formula (2.6) below we need additional notation and terminology.

\subsubsection{Pair partitions}

Let $\mathcal{F}_{n}$ denote the set of pair partitions (i.e., partitions into two-element sets) of the $2 n$-element set $\{ \pm 1, \pm 2, \ldots, \pm n\}$. Ref. [15] uses the notation $\{\hat{1}, 1, \hat{2}, 2, \ldots, \hat{n}, n\}$ for the same object - the ordered set of $2 n$ elements with the distinguished match. Other authors interpret $\mathcal{F}_{n}$ as the set of 1-factors, or perfect matchings, or 1-regular graphs on the vertices $\{ \pm 1, \pm 2, \ldots, \pm n\}$.

Identifying the pairs $\{i, j\}$ of a pair partition $\gamma \in \mathcal{F}_{n}$ with the cycles of the permutation, we can embed $\mathcal{F}_{n} \subset \mathcal{S}_{\{ \pm 1, \ldots, \pm n\}} \cong \mathcal{S}_{2 n}$. Here $\mathcal{S}_{F}$ denotes the group of permutations (i.e., bijections with composition) of a finite set F. As usual $\mathcal{S}_{n}=\mathcal{S}_{\{1, \ldots, n\}}$. In the embedding, $\mathcal{F}_{n}$ is the set of all involutions on $\{ \pm 1, \ldots, \pm n\}$ with no fixed points. Accordingly, we write $\gamma(i)=j$ if $\{i, j\} \in \gamma$.

We will also find it convenient to represent $\gamma$ as a graph with vertices arranged in two rows

$$
\begin{array}{rrrr}
1 & 2 & \ldots & n \\
-1 & -2 & \ldots & -n
\end{array}
$$

with the edges drawn between the vertices in each pair of partition $\gamma$. For example, a pair partition $\delta$ which pairs $j$ with $-j$ for $j=1, \ldots, n$ is identified with the permutation $\delta(j)=-j$, or with the graph

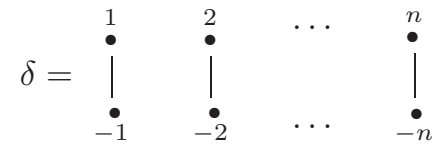

In fact, $\delta$ is the distinguished matching on $\{ \pm 1, \ldots, \pm n\}$ and plays a special role in several definitions that follow.

With each $\gamma \in \mathcal{F}_{n}$ we associate the permutation $\pi(\gamma) \in \mathcal{S}_{n}$ and the sequence $\varepsilon(\gamma)$ of \pm . These mappings $\pi: \mathcal{F}_{n} \rightarrow \mathcal{S}_{n}$ and $\varepsilon: \mathcal{F}_{n} \rightarrow\{-,+\}^{n}$ are defined in terms of graphs as follows.

Consider the 2-regular graph $\delta \cup \gamma$. To define the first cycle of permutation $\alpha:=\pi(\gamma)$, start with vertex -1 . Follow upwards the first edge of $\delta$ towards 1, and then continue by following the consecutive edges of the graph $\delta \cup \gamma$. The first cycle of $\alpha$ consists of the vertices from the upper row $\{1,2, \ldots, n\}$ that were visited by this path, taken in the order in which they were visited. The corresponding values of $\varepsilon(\gamma)$ on this cycle are determined by the direction of traversing the edges of $\delta$ : we assign + if the corresponding edge of $\delta$ was traversed upwards, and - otherwise. (In particular, the sequence $\varepsilon(\gamma)$ of signs always starts with + .)

The next cycle of $\pi(\gamma)$ consists of the entries in the first row, starting with the leftmost element $j$ of the first row that was has not yet been visited. To determine the cycle, we again start at the corresponding vertex $-j$ from the lower row, follow upwards the edge of $\delta$ towards $j$, and continue in the same direction along the edges of $\delta \cup \gamma$ until we complete the second cycle. We then repeat these steps until we exhaust all the elements of the first row. (Similar construction appears in Refs. [15, 17, 20.)

For example, suppose

$$
\gamma=\{\{1,2\},\{3,-4\},\{-1,-2\},\{-3,4\}\}=\stackrel{\bullet-\bullet}{\bullet} \bullet \chi^{\bullet}
$$


Then

$$
\delta \cup \gamma=i_{\bullet-\bullet}^{\bullet} \uparrow i_{\bullet}^{\bullet}
$$

Here the edges for the initial vertices of each cycle are oriented in the direction of the "first step". Thus $\pi(\gamma)=(1,2)(3,4)$ and $\varepsilon(\gamma)=(+,-,+,+)$.

Next, we introduce partitions that will play a role in Section 2.2

Example 2.1. For $\lambda \vdash n$, i.e. for $\lambda=\left(\lambda_{1} \geq \lambda_{2} \geq \cdots \geq 0\right)$ with $\sum \lambda_{j}=n$, consider

$$
\sigma_{\lambda}=\{\underbrace{\{1,-2\},\{2,-3\}, \ldots,\left\{\lambda_{1},-1\right\}}_{\lambda_{1} \text { pairs }}, \underbrace{\left\{\lambda_{1}+1,-\lambda_{1}-2\right\}, \ldots,\left\{\lambda_{1}+\lambda_{2},-\lambda_{1}-1\right\}}_{\lambda_{2} \text { pairs }}, \ldots\} .
$$

Then
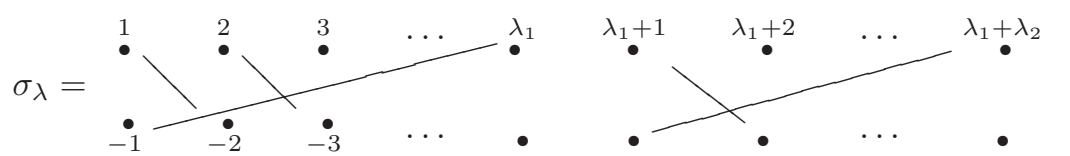

$\lambda_{1}+\lambda_{2}+1 \quad \cdots$

and
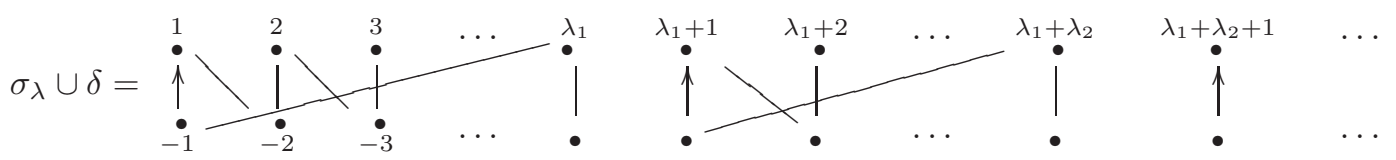

Thus $\pi\left(\sigma_{\lambda}\right)=\left(1,2, \ldots, \lambda_{1}\right)\left(\lambda_{1}+1, \ldots, \lambda_{1}+\lambda_{2}\right), \ldots$ has cycle type $\lambda$. In particular, $\delta=\sigma_{1^{n}}$ and $\pi(\delta)=i d$. Similarly, $\pi\left(\sigma_{n}\right)=(1,2, \ldots, n)$.

Definition 2.2. By $\mathcal{F}_{n}^{+}$we denote the set of pair partitions $\sigma \in \mathcal{F}_{n}$ in which the vertices of the upper row are always connected to vertices of the lower row. Such pair partitions are characterized by the fact that $\varepsilon=(+,+, \ldots,+)$.

Remark 2.3. Brauer [8] identifies the elements of $\mathcal{F}_{n}^{+}$with permutations, as $\pi: \mathcal{F}_{n}^{+} \rightarrow \mathcal{S}_{n}$ is a bijection. For $\sigma \in \mathcal{F}_{n}^{+}$, the definition of $\pi(\sigma)$ simplifies to

$$
\pi(\sigma)(j):=-\sigma(j), j=1,2, \ldots, n .
$$

The inverse mapping $\mathcal{S}_{n} \ni \alpha \mapsto \sigma \in \mathcal{F}_{n}^{+}$is then $\sigma(j)=-\alpha(j)$.

\subsubsection{Brauer product of pair partitions}

The Brauer product of pair partitions has simple description in terms of graphs. To define $\sigma$ $\odot$ we draw the two rows for $\gamma$, then we draw the graph for $\sigma$ so that its upper row covers the lower row of $\gamma$. We construct the new two-row graph for $\sigma \circ \gamma$ by removing the middle row, but retaining the edges between the vertices of the first and third row that were connected in the three-row graph. Brauer [8] weights the resulting graph by a coefficient that depends on the number of cycles lost in the middle row; we will apply the Brauer product only when there are no such cycles, so our weight will always be 1.

Example 2.4. Suppose

$$
\begin{aligned}
& \sigma=\{\{1,-2\},\{2,-3\},\{3,-4\},\{4,-1\}\}= \\
& \gamma=\{\{1,2\},\{3,-4\},\{-1,-2\},\{-3,4\}\}=
\end{aligned}
$$

The intermediate three-row graph is

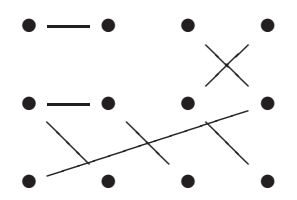


This gives

$$
\sigma \odot \gamma=\left.\underset{\bullet-\bullet}{\bullet}\right|_{\bullet} ^{\bullet}=\{\{1,2\},\{-2,-3\},\{-1,3\},\{-4,4\}\} .
$$

Our formulas need only the Brauer products with $\sigma \in \mathcal{F}_{n}^{+}$, and in this case the analytical definition takes the following form.

Definition 2.5. For $\sigma \in \mathcal{F}_{n}^{+}$and $\gamma \in \mathcal{F}_{n}$, the Brauer product [8] $\sigma \odot \gamma$ is defined as an element of $\mathcal{F}_{n}$ such that

$$
(\sigma \circ \gamma)(j)= \begin{cases}\sigma(-\gamma(j)) & \text { if } j>0, \gamma(j)<0, \\ \gamma(j) & \text { if } j>0, \gamma(j)>0, \\ \sigma(-\gamma(-\sigma(j))) & \text { if } j<0, \gamma(-\sigma(j))<0 \\ \gamma(-\sigma(j)) & \text { if } j<0, \gamma(-\sigma(j))>0\end{cases}
$$

(These are the same cases that are listed in Table 2])

Remark 2.6 (Brauer). If $\sigma_{1}, \sigma_{2} \in \mathcal{F}_{n}^{+}$then $\sigma_{1} \odot \sigma_{2} \in \mathcal{F}_{n}^{+}$and

$$
\pi\left(\sigma_{1} \odot \sigma_{2}\right)=\pi\left(\sigma_{1}\right) \circ \pi\left(\sigma_{2}\right)
$$

becomes just the ordinary composition of permutations.

We also remark that $\delta \odot \gamma=\gamma$ and, when the more general definition is used, $\gamma \odot \delta=\gamma$.

It will be convenient to have concise notation for certain objects and properties of $\gamma \in \mathcal{F}_{n}$ which depend only through the associated permutation $\pi(\gamma) \in \mathcal{S}_{n}$.

Definition 2.7. For a "coloring" $t:\{1, \ldots, n\} \rightarrow\{1, \ldots s\}$, by

$$
\mathcal{F}_{n}(t)=\left\{\gamma \in \mathcal{F}_{n}: t(|\gamma(j)|)=t(|j|), j= \pm 1, \ldots, \pm n\right\}
$$

we denote the set of color-preserving pair partitions. Such partitions are characterized by the property that the permutation $\alpha:=\pi(\gamma)$ is color-preserving, $t \circ \alpha=t$, see [9, (4)].

By $\mathcal{C}(\gamma)$ we denote the set of cycles of permutation $\pi(\gamma)$. For $\gamma \in \mathcal{F}_{n}(t)$, all cycles have the same color; by $\mathcal{C}_{j}(\gamma)$ we denote the set of cycles of $\pi(\gamma)$ which are of color $j \in\{1, \ldots, s\}$.

For a $\gamma \in \mathcal{F}_{n}(t)$, we can color each edge of $\gamma$ by the color of the adjacent vertices. Since for $\sigma \in \mathcal{F}_{n}^{+}$each edge of $\sigma \odot \gamma$ arises from combining exactly one edge of $\gamma$ with some of the edges of $\sigma$, we can color each edge of $\sigma \odot \gamma$ by the color inherited from this unique edge of $\gamma \in \mathcal{F}_{n}(t)$.

Definition 2.8. For $\gamma \in \mathcal{F}_{n}(t), \sigma \in \mathcal{F}_{n}^{+}$, by $t(\sigma, \gamma)$ we denote the coloring of $\{1,2, \ldots, n\}$ obtained as follows. For $1 \leq i \leq n$, we define $t(\sigma, \gamma)(i)$ as the color of the edge of $\sigma \odot \gamma$ which is crossed on $\delta \cup(\sigma \odot \gamma)$ while connecting $i$ to the next element of the corresponding cycle of $\pi(\sigma \odot \gamma)$.

\subsubsection{Formula for moments}

For a "coloring" $t:\{1, \ldots, n\} \rightarrow\{1, \ldots s\}$, a pair partition $\gamma \in \mathcal{F}_{n}$, and matrix-variables $\mathbf{x}_{1}, \mathbf{x}_{2}, \ldots, \mathbf{x}_{s}$, define monomial

$$
p_{\gamma, t}\left(\mathbf{x}_{1}, \mathbf{x}_{2}, \ldots, \mathbf{x}_{s}\right)=\prod_{c \in \mathcal{C}(\gamma)} \operatorname{tr}\left(\prod_{j \in c} \mathbf{x}_{t(j)}\right)
$$

Here the notation $\operatorname{tr}\left(\prod_{j \in c} \mathbf{x}_{t(j)}\right)$ is to be interpreted as the trace of the product of the matrices taken in the same order in which consecutive integers appear in the cycle $c$.

For example, $\operatorname{tr}^{2}\left(\mathbf{x}_{1} \mathbf{x}_{2}\right)=p_{\sigma, t}\left(\mathbf{x}_{1}, \mathbf{x}_{2}\right)$ with

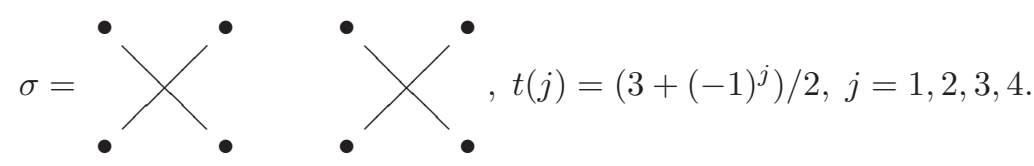

Clearly, such representation is not unique, as we can realize the same polynomial $p_{\sigma, t}$ by combining an appropriate mapping $t$ with any element $\gamma \in \mathcal{F}_{n}$ such that $\pi(\sigma)$ and $\pi(\gamma)$ are in the same conjugacy class. The generic 
construction is to start with a partition $\lambda \vdash n$, choose $\sigma_{\lambda} \in \mathcal{F}_{n}^{+}$according to (2.2), and then define a suitable function $t$. This generic construction indicates that the monomials $p_{\gamma, t}$ are essentially the monomials that appear in [9, Theorem 2], with $h \equiv I$.

Identifying $\mathbf{x}^{+}$with $\mathbf{x}$ and $\mathbf{x}^{-}$with the transpose $\mathbf{x}^{\prime}$ of a square matrix $\mathbf{x}$, define

$$
q_{\gamma, t}\left(\mathbf{x}_{1}, \mathbf{x}_{2}, \ldots, \mathbf{x}_{s}\right)=\prod_{c \in \mathcal{C}(\gamma)} \operatorname{tr}\left(\prod_{j \in c} \mathbf{x}_{t(j)}^{-\varepsilon_{j}(\gamma)}\right)
$$

Of course, if matrix variables $\mathbf{x}_{1}, \ldots, \mathbf{x}_{s}$ are symmetric, then $q_{\gamma, t}\left(\mathbf{x}_{1}, \mathbf{x}_{2}, \ldots, \mathbf{x}_{s}\right)=p_{\gamma, t}\left(\mathbf{x}_{1}, \mathbf{x}_{2}, \ldots, \mathbf{x}_{s}\right)$. Trivially, for general matrix variables the polynomials coincide when $\gamma \in \mathcal{F}_{n}^{+}$.

With the above notation, we state the main result of this section.

Theorem 2.9. Let $B_{1}, \ldots, B_{s} \in \mathcal{M}_{M \times M}$ and let $\Sigma_{1}, \ldots, \Sigma_{s}$ be $N \times N$ positive definite matrices. If $\mathbf{W}_{1} \in$ $\mathcal{W}\left(\Sigma_{1}, B_{1}\right), \mathbf{W}_{2} \in \mathcal{W}\left(\Sigma_{2}, B_{2}\right), \ldots, \mathbf{W}_{s} \in \mathcal{W}\left(\Sigma_{s}, B_{s}\right)$ are independent and $\sigma \in \mathcal{F}_{n}^{+}$, then

$$
\mathbb{E}\left(p_{\sigma, t}\left(\mathbf{W}_{1}, \mathbf{W}_{2}, \ldots, \mathbf{W}_{s}\right)\right)=\sum_{\gamma \in \mathcal{F}_{n}(t)} q_{\gamma, t}\left(B_{1}, \ldots, B_{s}\right) p_{\sigma \odot \gamma, t(\sigma, \gamma)}\left(\Sigma_{1}, \Sigma_{2}, \ldots, \Sigma_{s}\right)
$$

We postpone the proof to Section 3. In Section 2.2 we give a noncommutative generalization. This section concludes with examples, remarks and corollaries.

\subsubsection{Example of mean and variance calculation}

The purpose of this section is to illustrate (2.9) and also to show how the coloring $t(\sigma, \gamma)$ works in a simple case.

Example 2.10. Under the assumptions of Theorem [2.9, formula (2.9) gives

$$
\mathbb{E}\left(\operatorname{tr}\left(\mathbf{W}_{1} \mathbf{W}_{2} \ldots \mathbf{W}_{s}\right)\right)=\operatorname{tr}\left(B_{1}\right) \operatorname{tr}\left(B_{2}\right) \ldots \operatorname{tr}\left(B_{s}\right) \operatorname{tr}\left(\Sigma_{1} \Sigma_{2} \ldots \Sigma_{s}\right)
$$

Indeed, with $\sigma=\{(1,-2),(2,-3),(s-1,-s),(s,-1)\}$ and $t(j)=j$, we get $\mathcal{F}_{s}(t)=\{\delta\}$. Since $\sigma \odot \delta=\sigma$ and $t(\sigma, \delta)=t$, we get

$$
p_{\sigma \odot \gamma, t(\sigma, \gamma)}\left(\mathbf{x}_{1}, \ldots, \mathbf{x}_{s}\right)=p_{\sigma, t}\left(\mathbf{x}_{1}, \ldots, \mathbf{x}_{s}\right)=\operatorname{tr}\left(\mathbf{x}_{1} \ldots \mathbf{x}_{s}\right)
$$

and

$$
q_{\delta, t}\left(\mathbf{x}_{1}, \ldots, \mathbf{x}_{s}\right)=\operatorname{tr}\left(\mathbf{x}_{1}^{\prime}\right) \ldots \operatorname{tr}\left(\mathbf{x}_{s}^{\prime}\right)=\operatorname{tr}\left(\mathbf{x}_{1}\right) \ldots \operatorname{tr}\left(\mathbf{x}_{s}\right) .
$$

Example 2.11. Suppose $\mathbf{W}_{1}, \mathbf{W}_{2}$ are as in Theorem 2.9. From Example 2.10 we know that $\mathbb{E}\left(\operatorname{tr}\left(\mathbf{W}_{1} \mathbf{W}_{2}\right)\right)=$ $\operatorname{tr}\left(B_{1}\right) \operatorname{tr}\left(B_{2}\right) \operatorname{tr}\left(\Sigma_{1} \Sigma_{2}\right)$. To compute the variance of $\operatorname{tr}\left(\mathbf{W}_{1} \mathbf{W}_{2}\right)$ we therefore compute $\mathbb{E}\left(\operatorname{tr}^{2}\left(\mathbf{W}_{1} \mathbf{W}_{2}\right)\right)$ using (2.9) for $n=4$ with $\sigma, t$ as defined in (2.7). Nine possible $\gamma \in \mathcal{F}_{4}(t)$ and their contributions are listed in Table 1. Thus

$$
\begin{aligned}
\operatorname{Var}\left(\mathbf{W}_{1} \mathbf{W}_{2}\right)=( & \left.\operatorname{tr}\left(B_{1} B_{1}^{\prime}\right) \operatorname{tr}\left(B_{2} B_{2}^{\prime}\right)+\operatorname{tr}\left(B_{1}^{2}\right) \operatorname{tr}\left(B_{2}^{2}\right)\right) \operatorname{tr}^{2}\left(\Sigma_{1} \Sigma_{2}\right) \\
& +\left(\operatorname{tr}\left(B_{1} B_{1}^{\prime}\right) \operatorname{tr}\left(B_{2}^{2}\right)+\operatorname{tr}\left(B_{1}^{2}\right) \operatorname{tr}\left(B_{2} B_{2}^{\prime}\right)+\operatorname{tr}^{2}\left(B_{1}\right) \operatorname{tr}\left(B_{2}^{2}\right)+\operatorname{tr}^{2}\left(B_{1}\right) \operatorname{tr}\left(B_{2} B_{2}^{\prime}\right)\right. \\
& \left.+\operatorname{tr}\left(B_{1}^{2}\right) \operatorname{tr}^{2}\left(B_{2}\right)+\operatorname{tr}\left(B_{1} B_{1}^{\prime}\right) \operatorname{tr}^{2}\left(B_{2}\right)\right) \operatorname{tr}\left(\left(\Sigma_{1} \Sigma_{2}\right)^{2}\right)
\end{aligned}
$$

With $B_{j}=\left[\begin{array}{cc}I_{M_{j}} & 0 \\ 0 & 0\end{array}\right]$ and $\Sigma_{j}=I_{N}$ this gives

$$
\operatorname{Var}\left(\mathbf{W}_{1} \mathbf{W}_{2}\right)=2 M_{1} M_{2} N^{2}+2 M_{1} M_{2}\left(M_{1}+M_{2}+1\right) N
$$

(See also Example 4.8.)

\subsubsection{Remarks}

Remark 2.12. Wishart expansions can also be modeled by planar algebras, see [19. 
Table 1: Terms in (2.9) for calculating $\mathbb{E}\left(\operatorname{tr}^{2}\left(\mathbf{W}_{1} \mathbf{W}_{2}\right)\right)$; here $\sigma$ is given by (2.7). The second column is an illustration for Section 2.2 .

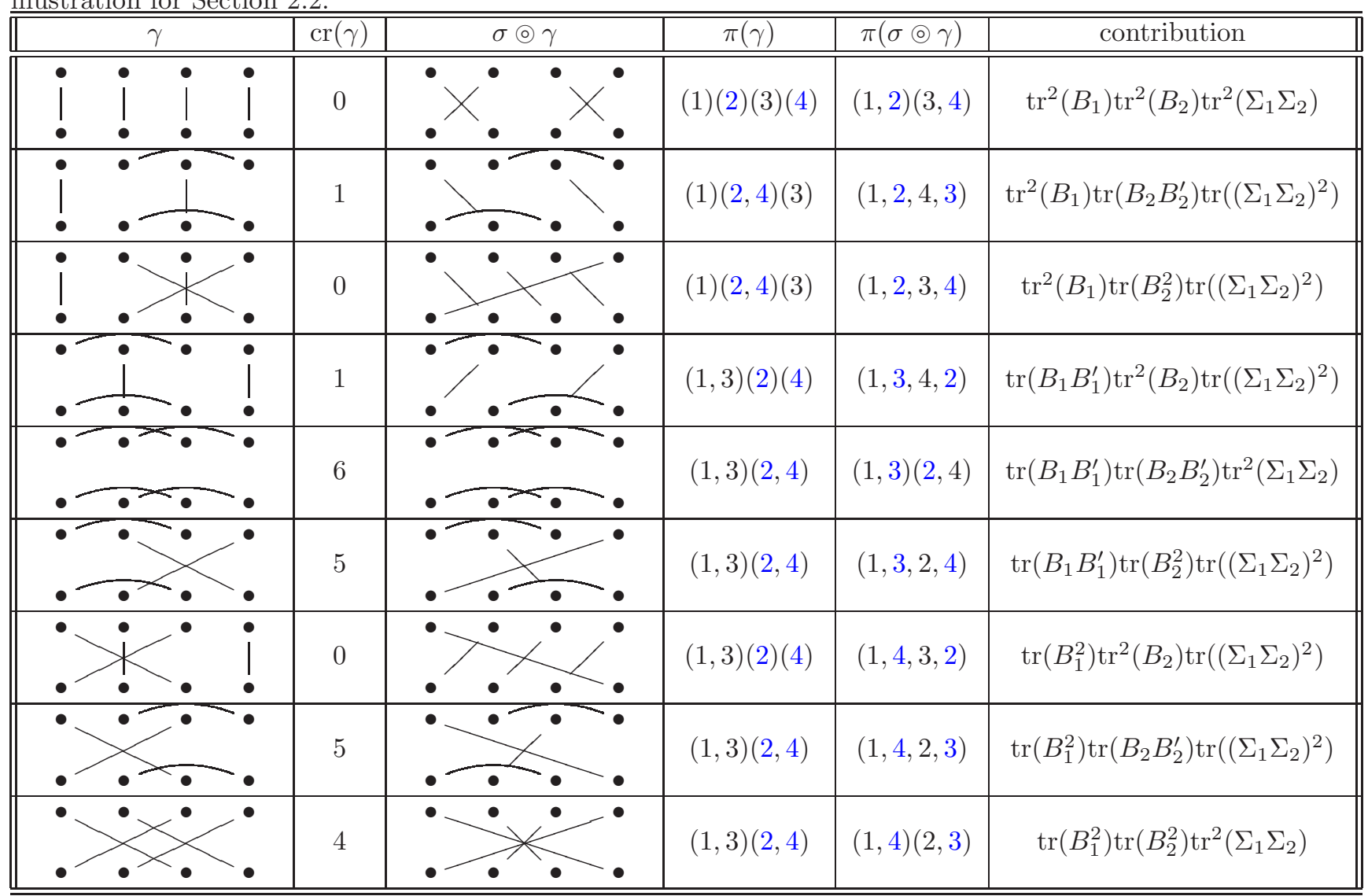

Remark 2.13. Applying (1.1) to complex Gaussian X, one can define the corresponding "generalized complex Wishart" matrices with complex-valued $B$ and Hermitian $\Sigma$. [9, Theorem 2] can be generalized to this setting, and the resulting formula is

$$
\mathbb{E}\left(p_{\sigma, t}\left(\mathbf{W}_{1}, \mathbf{W}_{2}, \ldots, \mathbf{W}_{s}\right)\right)=\sum_{\gamma \in \mathcal{F}_{n}^{+}(t)} p_{\gamma^{-1}, t}\left(B_{1}, \ldots, B_{s}\right) p_{\sigma \odot \gamma, t}\left(\Sigma_{1}, \Sigma_{2}, \ldots, \Sigma_{s}\right) .
$$

Moreover, since $\gamma, \sigma \in \mathcal{F}_{n}^{+}$, we can identify them with the pairs of permutations $\pi(\gamma)$ and $\pi(\sigma)$ respectively; this identification does not affect monomials $p_{\gamma}$ which depend only on the cycle type of permutation $\pi(\gamma)$. When $\varepsilon=(+, \ldots,+)$, we have $q_{\gamma}=p_{\gamma}$, and $t(\sigma, \gamma)=t$; since the identification maps Brauer product to composition of permutations, see (2.5), we can write this formula directly in terms of permutations:

$$
\mathbb{E}\left(p_{\sigma, t}\left(\mathbf{W}_{1}, \mathbf{W}_{2}, \ldots, \mathbf{W}_{s}\right)\right)=\sum_{\gamma \in \mathcal{S}_{n}(t)} p_{\gamma^{-1}, t}\left(B_{1}, \ldots, B_{s}\right) p_{\sigma \circ \gamma, t}\left(\Sigma_{1}, \Sigma_{2}, \ldots, \Sigma_{s}\right)
$$

Proof. Indeed, in the complex case the only pairings $\gamma$ that contribute to Wick formula are $\gamma \in \mathcal{F}_{n}^{+}$, so the proof of (2.9) gives

$$
\mathbb{E}\left(p_{\sigma, t}\left(\mathbf{W}_{1}, \mathbf{W}_{2}, \ldots, \mathbf{W}_{s}\right)\right)=\sum_{\gamma \in \mathcal{F}_{n}^{+}(t)} q_{\gamma, t}\left(B_{1}^{\prime}, \ldots, B_{s}^{\prime}\right) p_{\sigma \odot \gamma, t}\left(\Sigma_{1}, \Sigma_{2}, \ldots, \Sigma_{s}\right)
$$

Then we note that with $*$ denoting the conjugate transpose, we have $\operatorname{tr}(A B \ldots C)=\overline{\operatorname{tr}\left(C^{*} \ldots B^{*} A^{*}\right)}$, so if $c \in \mathcal{C}(\gamma)$ then

$$
\operatorname{tr}\left(\prod_{j \in c} B_{t(j)}^{\prime}\right)=\overline{\operatorname{tr}\left(\prod_{j \in c^{-1}}\left(B_{t(j)}^{\prime}\right)^{*}\right)}=\overline{\operatorname{tr}\left(\prod_{j \in c^{-1}} \bar{B}_{t(j)}\right)}=\operatorname{tr}\left(\prod_{j \in c^{-1}} B_{t(j)}\right) .
$$




\subsubsection{Special cases of interest}

In this section we list special cases of Theorem 2.9. Several similar one-matrix cases appeared in the literature, but without the use of the Brauer product the formulas may look differently at first.

The following specialization of Theorem 2.9 shows that in the real-Wishart case the Brauer plays a role analogous to the composition of permutations for the complex-Wishart case in [9, Theorem 2].

Corollary 2.14. If $\mathbf{W}_{1} \in \mathcal{W}\left(\Sigma_{1}, I_{M_{1}}\right), \mathbf{W}_{2} \in \mathcal{W}\left(\Sigma_{2}, I_{M_{2}}\right), \ldots, \mathbf{W}_{s} \in \mathcal{W}\left(\Sigma_{s}, I_{M_{s}}\right)$ are independent and $\sigma \in$ $\mathcal{F}_{n}^{+}$, then

$$
\mathbb{E}\left(p_{\sigma, t}\left(\mathbf{W}_{1}, \mathbf{W}_{2}, \ldots, \mathbf{W}_{s}\right)\right)=\sum_{\gamma \in \mathcal{F}_{n}(t)} \prod_{j=1}^{s} M_{j}^{\# \mathcal{C}_{j}(\gamma)} p_{\sigma \odot \gamma, t(\sigma, \gamma)}\left(\Sigma_{1}, \Sigma_{2}, \ldots, \Sigma_{s}\right)
$$

Proof. Let $M=\max \left\{M_{1}, \ldots, M_{s}\right\}$. We apply Theorem 2.9 to $M \times M$ matrices

$$
B_{j}=\left[\begin{array}{cc}
I_{M_{j}} & 0 \\
0 & 0
\end{array}\right]
$$

Since the cycles of $\pi(\gamma)$ preserve $t$, a cycle of color $r$ contributes one factor $\operatorname{tr}\left(B_{r}\right)=M_{r}$ to $q_{\gamma, t}\left(B_{1}, \ldots, B_{s}\right)$. Thus $q_{\gamma, t}\left(B_{1}, \ldots, B_{s}\right)=\prod_{j=1}^{s} M_{j}^{\# \mathcal{C}_{j}(\gamma)}$.

Remark 2.15. For a single real Wishart matrix, Graczyk, Letac \& Massam [17] compute moments of more general monomials that involve additional arbitrary matrix parameters. It is plausible that Corollary 2.14 can be deduced from their result by consecutive integration with respect to some of the auxiliary matrix parameters.

The following one-matrix case of Theorem 2.9] is a version of [20, Theorem 3.5], who did not use the Brauer product explicitly. (In the one-matrix case $t \equiv 1$ and $q_{\gamma, t}=p_{\gamma, t}$, so we will write $p_{\gamma}$ for both polynomials.)

Corollary 2.16. If $\mathbf{W} \in \mathcal{W}(\Sigma, B), B$ is symmetric, and $\sigma \in \mathcal{F}_{n}^{+}$, then

$$
\mathbb{E}\left(p_{\sigma}(\mathbf{W})\right)=\sum_{\gamma \in \mathcal{F}_{n}(t)} p_{\gamma}(B) p_{\sigma \odot \gamma}(\Sigma)
$$

To compute $p_{\gamma}\left(I_{N}\right)$ one only needs to count the number of cycles in $\gamma \in \mathcal{F}_{n}$. So when $\Sigma, B$ are both identity matrices, the expression depends only on the number of cycles of the appropriate 2-regular graphs.

Corollary 2.17 ([20, Corollary 2.4]). For $\lambda=\left(\lambda_{1}, \lambda_{2}, \ldots\right) \vdash n$, let $p_{\lambda}(\mathbf{x})=\operatorname{tr}\left(\mathbf{x}^{\lambda_{1}}\right) \operatorname{tr}\left(\mathbf{x}^{\lambda_{2}}\right) \ldots$ If $\mathbf{W} \in \mathcal{W}\left(I_{N}, I_{M}\right)$, and $\sigma_{\lambda} \in \mathcal{F}_{n}^{+}$is given by (2.2), then

$$
\mathbb{E}\left(p_{\lambda}(\mathbf{W})\right)=\sum_{\gamma \in \mathcal{F}_{n}} M^{\# \mathcal{C}(\delta \cup \gamma)} N^{\# \mathcal{C}\left(\gamma \cup \sigma_{\lambda}\right)} .
$$

Proof. From the definition of $\pi(\gamma)$ it is clear that the number of cycles of $\delta \cup \gamma$ matches the number of cycles of $\pi(\gamma)$.

The cycles of $\pi(\sigma \odot \gamma)$ arise from adding the vertical edges to connect the first and last row of the three-row diagram. In view of our choice of $\sigma_{\lambda}$, this gives the same cycles as adding to the graph of $\gamma$ the edges from the graph of $\hat{\sigma}$ defined by $\hat{\sigma}_{\lambda}(i)=-\sigma_{\lambda}(-i)$, which is just the graph of $\sigma_{\lambda}$ with swapped rows. Of course $\pi\left(\hat{\sigma}_{\lambda}\right)$ is the inverse of $\pi\left(\sigma_{\lambda}\right)$ so it has the same cycle structure. Thus $p_{\lambda}=p_{\hat{\sigma}_{\lambda}}$. Applying (2.9) to $p_{\hat{\sigma}_{\lambda}}$, we end with (2.13).

\subsection{Noncommutative version}

To extend Theorem 2.9 to noncommutative setting we need additional notation.

For $\gamma \in \mathcal{F}_{n}$, let $\operatorname{cr}(\gamma)$ denote the number of crossings of $\gamma$ treated as the pair partition of the ordered set $(1,-1,2,-2, \ldots, n,-n)$. (The second column of Table 1 lists some examples.)

In order to extend the definition of polynomials $p_{\sigma, t}$ to matrix arguments with entries that do not commute we need to specify the order in which the traces appear. To do so, we follow the standard conventions:

- we write the cycles of permutation $\pi(\sigma)$ starting with the smallest element. Thus if $c_{k}=\left(j_{k 1}, j_{k 2}, \ldots, j_{k \ell_{k}}\right)$ is the $k$-th cycle, then we assume that $j_{k 1} \leq j$ for all $j \in c_{k}$.

- we order the cycles $c_{1}, c_{2}, \ldots$ by the order of their first elements. Thus $1=j_{11}<j_{21}<\ldots$ 
Under these conventions, we define

$$
p_{\sigma, t}\left(\mathbf{W}_{1}, \ldots, \mathbf{W}_{s}\right)=\operatorname{tr}\left(\mathbf{W}_{t(1)} \mathbf{W}_{t\left(j_{12}\right)} \mathbf{W}_{t\left(j_{1 \ell_{1}}\right)}\right) \operatorname{tr}\left(\mathbf{W}_{t\left(j_{21}\right)} \mathbf{W}_{t\left(j_{22}\right)} \ldots \mathbf{W}_{t\left(j_{2 \ell_{2}}\right)}\right) \ldots
$$

We will use shorthand notation

$$
p_{\sigma, t}\left(\mathbf{W}_{1}, \ldots, \mathbf{W}_{s}\right)=\prod_{c \in \mathcal{C}(\sigma)} \operatorname{tr}\left(\prod_{j \in c} \mathbf{W}_{t(j)}\right)
$$

which should be interpreted in the above sense.

Our next result extends Theorem 2.9 to noncommutative setting. We remark that due to noncommutativity, we allow only special choices of $\sigma$, and that polynomials $q_{\gamma, t}$ are not needed, as matrices $B_{1}, \ldots, B_{s}$ are symmetric.

Theorem 2.18. Let $B_{1}, \ldots, B_{s} \in \mathcal{M}_{M \times M}(\mathbb{R})$ and $\Sigma_{1}, \ldots, \Sigma_{s} \in \mathcal{M}_{N \times N}(\mathbb{R})$ be positive definite matrices. Let $\sigma \in \mathcal{F}_{n}^{+}$be given by (2.2) and let $t:\{1, \ldots, n\} \rightarrow\{1, \ldots, s\}$. If $\mathbf{W}_{1} \in \mathcal{W}_{q}\left(\Sigma_{1}, B_{1}\right), \mathbf{W}_{2} \in \mathcal{W}_{q}\left(\Sigma_{2}, B_{2}\right), \ldots, \mathbf{W}_{s} \in$ $\mathcal{W}_{q}\left(\Sigma_{s}, B_{s}\right)$ are q-orthogonal, $-1 \leq q \leq 1$, then

$$
\tau\left(p_{\sigma, t}\left(\mathbf{W}_{1}, \ldots, \mathbf{W}_{s}\right)\right)=\sum_{\gamma \in \mathcal{F}_{n}(t)} q^{\mathrm{cr}(\gamma)} p_{\gamma, t}\left(B_{1}, \ldots, B_{s}\right) p_{\sigma \odot \gamma, t(\sigma, \gamma)}\left(\Sigma_{1}, \Sigma_{2}, \ldots, \Sigma_{s}\right) .
$$

The proof is given in Section 3 . Here we apply (2.14) to deduce a non-asymptotic representation of the Marchenko-Pastur law.

\subsubsection{A matrix representation of the compound Marchenko-Pastur law}

There is a number of results that relate Marchenko-Pastur law to the semicircle law. The well known result from [37] says that if $\mathbf{X}$ has the semicircle law then $\mathbf{X}^{2}$ has the Marchenko-Pastur law with parameter $\lambda=1$. (In fact, Ref. 37] shows that for every value of $\lambda>0$, one can find a projection $P$ such that $\mathbf{X} P \mathbf{X}$ has the Marchenko-Pastur law.) A related representation arises by interpreting an $n M \times n N$ matrix of independent random variables as an $M \times N$ block matrix; the limit as $n \rightarrow \infty$ represents the Marchenko-Pastur law with parameter $M / N$ as $\mathbf{X}^{*} \mathbf{X}$ with a matrix $\mathbf{X}$ of free circular entries. Yet another representation that uses free circular elements appears in [43, Proposition 5.2].

Our representation of the Marchenko-Pastur law differs slightly, as our matrix $\mathbf{X}$ is not self-adjoint and has semicircular entries. Of course, the result is not surprising; but an argument is needed as occasionally squares of symmetric and nonsymmetric matrices behave differently, see [34, Section 4].

We first recall some properties of non-crossing partitions and recall the definition of a compound MarchenkoPastur law.

A partition $\mathcal{V}$ of an ordered set is non-crossing if $\operatorname{cr}(\mathcal{V})=0$. By $N C[1, \ldots, n]$ we denote the set of all non-crossing partitions of $(1, \ldots, n)$.

The free cumulants $\left\{r_{k}: k \geq 1\right\}$ of a probability measure $\mu$ with all moments are defined implicitly by requiring that for all $n \in \mathbb{N}$,

$$
\int_{\mathbb{R}} x^{n} \mu(d x)=\sum_{\mathcal{V} \in N C[1, \ldots, n]} \prod_{V \in \mathcal{V}} r_{\# V} .
$$

For more details, see [21, Section 2.5]; for the operator-valued case, see [41.

The Marchenko-Pastur law with parameter $\lambda>0$ is the law

$$
\pi_{\lambda}(d x)=(1-\lambda)^{+} \delta_{0}+\frac{\sqrt{4 \lambda-(x-1-\lambda)^{2}}}{2 \pi x} 1_{(1-\sqrt{\lambda})^{2} \leq x \leq(1+\sqrt{\lambda})^{2}} d x
$$

where $\delta_{0}$ denotes the point mass at 0 . The free cumulants of the Marchenko-Pastur law are $r_{k}=\lambda$ for all $k \geq 1$.

If $\nu$ is a probability measure on the Borel sets of $\mathbb{R}$ with finite moments, then the compound MarchenkoPastur law $\pi_{\nu, \lambda}(d x)$ is defined as the law with free cumulants

$$
r_{k}=\lambda \int_{\mathbb{R}} x^{k} \mu(d x) .
$$

Thus the $n$-th moment of the compound Marchenko-Pastur law is

$$
\int_{\mathbb{R}} x^{n} \pi_{\nu, \lambda}(d x)=\sum_{\mathcal{V} \in N C[1 \ldots n]} \lambda^{\# \mathcal{V}} \prod_{V \in \mathcal{V}} \int_{\mathbb{R}} x^{\# V} \mu(d x),
$$

compare [21, (3.3.10)]. In particular, the Marchenko-Pastur law corresponds to $\nu=\delta_{1}$. 
Proposition 2.19. Let $\nu$ be the distribution of the eigenvalues of a real positive-definite matrix $B$ with respect to the normalized $\operatorname{tr}_{M}: \mathcal{M}_{M \times M} \rightarrow \mathbb{R}$. (That is, $\nu$ assigns mass $1 / M$ to each of the eigenvalues of $B$ ). Let $\lambda=M / N$. If $\mathbf{W} \in \mathcal{W}_{q}\left(I_{N}, B\right)$ and $q=0$ then $\frac{1}{N} \mathbf{W}$ has compound Marchenko-Pastur law $\pi_{\nu, \lambda}$ with respect to state $\tau \circ \operatorname{tr}_{N}$. In particular, if $\mathbf{W} \in \mathcal{W}_{q}\left(I_{N}, I_{M}\right)$ and $q=0$ then $\frac{1}{N} \mathbf{W}$ has Marchenko-Pastur law with parameter $\lambda$.

A pair partition $\gamma \in \mathcal{F}_{n}$ is non-crossing, if $\operatorname{cr}(\gamma)=0$. Notice that all non-crossing $\gamma$ are in $\mathcal{F}_{n}^{+}$. Indeed, our ordering of $\{1,-1, \ldots, n,-n\}$ implies that any two numbers of the same sign have an odd number of elements between them. Thus any pair in $\gamma$ of the same sign must generate a crossing.

We will need to know that for a non-crossing $\gamma \in \mathcal{F}_{n}$, permutation $\pi(\gamma)$ is the inverse of a non-crossing permutation. Biane [3] defines non-crossing permutations $\beta \in \mathcal{S}_{n}$ by the following two requirements:

(a) Each cycle of $\beta$ can be written in increasing order

(b) The partition of $\{1, \ldots, n\}$ into the cycles of $\beta$ is non-crossing.

Biane characterizes non-crossing permutations by the geodesic condition

$$
\# \mathcal{C}(\beta)+\# \mathcal{C}\left(\rho \circ \beta^{-1}\right)=n+1
$$

(See [33, Remark 2.9], 25, Section 6], and [42, Exercise 6.19.hh]. Note that this is $g=0$ in (4.3) below.)

It is well known that the non-crossing pair partitions of $2 n$ elements are in a one-to-one correspondence with the non-crossing partitions of $n$ elements, see [42, Exercise 6.19]; see also [21, page 74]. It turns out that the restriction of $\pi: \mathcal{F}_{n} \rightarrow \mathcal{S}_{n}$ to non-crossing pair partitions defines an explicit version of this bijection.

Lemma 2.20. Let $\tilde{\pi}$ be the mapping from pair partitions of $\{ \pm 1, \ldots, \pm n\}$ to partitions of $\{1, \ldots, n\}$ which assigns to $\gamma \in \mathcal{F}_{n}$ the partition into the cycles of permutation $\pi(\gamma)$. Then $\tilde{\pi}$ is a bijection from the non-crossing pair partitions with respect to ordering $(1,-1, \ldots, n,-n)$ to the non-crossing partitions of $(1, \ldots, n)$ in natural order. Moreover, for non-crossing $\gamma \in \mathcal{F}_{n}$, all cycles of $\pi(\gamma)$ can be represented as decreasing sequences.

(We note that our "standard" representation of a cycle begins with the smallest element, which therefore needs to be moved to the end of the cycle to get a decreasing sequence representation.)

The proof below is due to the referee.

Proof. Non-crossing pair partitions are in $\mathcal{F}_{n}^{+}$, and we already noted in Remark 2.3 that $\pi: \mathcal{F}_{n}^{+} \rightarrow \mathcal{S}_{n}$ is one-to-one.

Let $\gamma \in \mathcal{F}_{n}^{+}$and let $\alpha \in \mathcal{S}$ be such that $\pi(\gamma)=\alpha$. Notice that permutation $\gamma \circ(1,-1,2,-2, \ldots, n,-n)$ works by sending $i \mapsto \alpha^{-1}(i)$ and $-i \mapsto-(\alpha \circ(1,2, \ldots, n))$, for every $1 \leq i \leq n$. Hence

$$
\# \mathcal{C}(\gamma \circ(1,-1,2,-2, \ldots, n,-n))=\# \mathcal{C}\left(\alpha^{-1}\right)+\# \mathcal{C}(\alpha \circ(1,2, \ldots, n))
$$

By using this fact and Biane's geodesic condition (2.16), we see that $\gamma$ is non-crossing with respect to $(1,-2,2,-2, \ldots, n,-n)$ iff

$$
\# \mathcal{C}(\gamma)+\# \mathcal{C}(\gamma \circ(1,-1,2,-2, \ldots, n,-n))=2 n+1
$$

Since $\# \mathcal{C}(\gamma)=n$, from (2.17) we see that this is equivalent to

$$
\# \mathcal{C}\left(\alpha^{-1}\right)+\# \mathcal{C}(\alpha \circ(1,2, \ldots, n))=n+1
$$

which, again by (2.16), is equivalent to the statement that $\alpha^{-1}$ is non-crossing.

Proof of Proposition 2.19. When $q=0$, 2.14 gives

$$
N^{-n} \tau \circ \operatorname{tr}_{N}\left(\mathbf{W}^{n}\right)=\sum_{\gamma} N^{\# \mathcal{C}(\sigma \odot \gamma)-n-1} \prod_{c \in \mathcal{C}(\gamma)} \operatorname{tr}\left(B^{\# c}\right)
$$

and the sum is taken over the non-crossing pair partitions $\gamma$. Non-crossing $\gamma$ are in $\mathcal{F}_{n}^{+}$, so we can use (2.5). With $\rho=\pi(\sigma)=(1, \ldots, n)$, we get

$$
N^{-n} \tau \circ \operatorname{tr}_{N}\left(\mathbf{W}^{n}\right)=\sum_{\gamma \in \mathcal{F}_{n}} N_{\text {non-crossing }}^{\# \mathcal{C}(\rho \circ \pi(\gamma))-n-1} \prod_{c \in \mathcal{C}(\gamma)} \operatorname{tr}\left(B^{\# c}\right) .
$$


By Lemma 2.20, we can take the sum over the permutations $\alpha$ obtained as images of non-crossing $\gamma$.

$$
N^{-n} \tau \circ \operatorname{tr}_{N}\left(\mathbf{W}^{n}\right)=\sum_{\alpha} N^{\# \mathcal{C}(\rho \circ \alpha)-n-1} \prod_{c \in \mathcal{C}(\alpha)} \operatorname{tr}\left(B^{\# c}\right)=\sum_{\alpha} \lambda^{\# \mathcal{C}(\alpha)} N^{\# \mathcal{C}(\alpha)+\# \mathcal{C}(\rho \circ \alpha)-n-1} \prod_{c \in \mathcal{C}(\alpha)} \operatorname{tr}_{M}\left(B^{\# c}\right)
$$

If the cycles of $\alpha \in \mathcal{S}_{n}$ form a non-crossing partition and are in decreasing order, then the cycles of $\beta:=\alpha^{-1}$ are in increasing order and form a non-crossing partition, too. Since $\alpha$ and $\alpha^{-1}$ have the same number of cycles, (2.16) can be written as

$$
\# \mathcal{C}(\rho \circ \alpha)+\# \mathcal{C}(\alpha)=n+1
$$

Thus,

$$
N^{-n} \tau \circ \operatorname{tr}_{N}\left(\mathbf{W}^{n}\right)=\sum_{\alpha \in \mathcal{S}_{n}, \alpha^{-1}} \lambda_{\text {non-crossing }}^{\# \mathcal{C}(\alpha)} \prod_{c \in \mathcal{C}(\alpha)} \int x^{\# c} \nu(d x)=\sum_{\mathcal{V} \in N C[1, \ldots, n]} \lambda^{\# \mathcal{V}} \prod_{V \in \mathcal{V}} \int x^{\# V} \nu(d x),
$$

which matches the moments (2.15) of the compound Marchenko-Pastur law.

\section{Proofs of Theorems 2.9 and 2.18}

Following [33], see [9. Lemma 1], we write polynomials $p_{\gamma, t}$ and $q_{\gamma, t}$ as follows.

Lemma 3.1. Let $\gamma \in \mathcal{F}_{n}$. With $\alpha=\pi(\gamma),\left(\varepsilon_{1}, \ldots \varepsilon_{n}\right)=\varepsilon(\gamma)$,

$$
\begin{aligned}
& p_{\gamma, t}\left(\mathbf{x}_{1}, \ldots, \mathbf{x}_{s}\right)=\sum_{I:\{1, \ldots, n\} \rightarrow\{1, \ldots, N\}} \prod_{i=1}^{n}\left[\mathbf{x}_{t(i)}\right]_{I(i), I(\alpha(i))}, \\
& q_{\gamma, t}\left(\mathbf{x}_{1}, \ldots, \mathbf{x}_{s}\right)=\sum_{I:\{1, \ldots, n\} \rightarrow\{1, \ldots, N\}} \prod_{i=1}^{n}\left[\mathbf{x}_{t(i)}^{-\varepsilon_{i}}\right]_{I(i), I(\alpha(i))} .
\end{aligned}
$$

Proof of Theorem [2.9. Denote $\rho=\pi(\sigma) \in \mathcal{S}_{n}$. Expanding matrix multiplications in (3.1), we write the polynomial on the left hand side of (2.9) as

$$
\begin{aligned}
& p_{\sigma, t}\left(\mathbf{W}_{1}, \mathbf{W}_{2}, \ldots, \mathbf{W}_{s}\right)=\sum_{I:\{1, \ldots, n\} \rightarrow\{1, \ldots, N\}} \prod_{i=1}^{n}\left[A_{t(i)}^{\prime} \mathbf{X}_{t(i)}^{\prime} B_{t(i)} \mathbf{X}_{t(i)} A_{t(i)}\right]_{I(i), I(\rho(i))} \\
& \quad=\sum_{I, U, K, J, V}\left(\prod_{i=1}^{n}\left[\mathbf{X}_{t(i)}^{\prime}\right]_{U(i), K(i)}\left[\mathbf{X}_{t(i)}\right]_{J(i), V(i)}\right) \prod_{i=1}^{n}\left[A_{t(i)}^{\prime}\right]_{I(i), U(i)}\left[A_{t(i)}\right]_{V(i), I(\rho(i))} \prod_{i=1}^{n}\left[B_{t(i)}\right]_{K(i), J(i)}
\end{aligned}
$$

where the sum is now taken over all functions $I, U, V:\{1, \ldots, n\} \rightarrow\{1, \ldots, N\}$ and over all functions $K, J:$ $\{1, \ldots, n\} \rightarrow\{1, \ldots, M\}$. The Wick formula expresses the expectation of the product of jointly normal centered random variables as the sum over all pairings of product of the covariances for each pair of random variables, compare Definition 1.2 with $q=1$. To describe the pairings, we associate positive integers $j=1,2, \ldots, n$ with $\mathbf{X}_{t(j)}^{\prime}$ and the negative integers $-j$ with $\mathbf{X}_{t(j)}$. Then the pairings are determined by $\gamma \in \mathcal{F}_{n}(t)$; denoting by $\alpha=\pi(\gamma)$, Table 2 lists four types of matches that might contribute to the moments.

The expectation becomes

$$
\mathbb{E}\left(\prod_{i=1}^{n}\left[\mathbf{X}_{t(i)}^{\prime}\right]_{U(i), K(i)}\left[\mathbf{X}_{t(i)}\right]_{J(i), V(i)}\right)=\sum_{\gamma \in \mathcal{F}_{n}(t)} \chi_{A}(\gamma, U, V) \chi_{B}(\gamma, K, J)
$$

where $\chi_{A}(\gamma, U, V) \chi_{B}(\gamma, K, J)=1$ only when functions $U, V$ and $K, J$, fulfill the matches specified in the last column of Table 2 at each edge of $\gamma$; otherwise, $\chi_{A}(\gamma, U, V) \chi_{B}(\gamma, K, J)=0$.

This allows us to write

$$
\mathbb{E}\left(p_{\sigma, t}\left(\mathbf{W}_{1}, \mathbf{W}_{2}, \ldots, \mathbf{W}_{s}\right)\right)=\sum_{\gamma \in \mathcal{F}_{n}(t)} \Pi_{A}(\gamma) \Pi_{B}(\gamma)
$$


Table 2: Types of matches along $\gamma \in \mathcal{F}_{n}(t)$; here $\alpha=\pi(\gamma) \in \mathcal{S}_{n}$.

\begin{tabular}{|c|c|c|c|}
\hline Match type & graph & matching entries & identification of indices \\
\hline $\mathrm{A}$ & $\stackrel{j}{\bullet} \stackrel{\alpha}{\bullet}_{\bullet}^{(j)}$ & {$\left[\mathbf{X}_{t(j)}^{\prime}\right]_{U(j) K(j)}=\left[\mathbf{X}_{t(\alpha(j))}\right]_{J(\alpha(j)) V(\alpha(j))}$} & $U(j)=V(\alpha(j)), K(j)=J(\alpha(j))$ \\
\hline B & $\begin{array}{r}j \\
\bullet \\
\bullet\end{array}$ & {$\left[\mathbf{X}_{t(j)}^{\prime}\right]_{U(j) K(j)}=\left[\mathbf{X}_{t(\alpha(j))}^{\prime}\right]_{U(\alpha(j)) K(\alpha(j))}$} & $U(j)=U(\alpha(j)), K(j)=K(\alpha(j))$ \\
\hline $\mathrm{C}$ & $\begin{array}{l}j \\
\bullet \\
\bullet\end{array}$ & {$\left[\mathbf{X}_{t(j)}\right]_{J(j) V(j)}=\left[\mathbf{X}_{t(\alpha(j))}\right]_{J(\alpha(j)) V(\alpha(j))}$} & $J(j)=J(\alpha(j)), V(j)=V(\alpha(j))$ \\
\hline$A^{-1}$ & $\bullet$ & {$\left[\mathbf{X}_{t(j)}\right]_{J(j) V(j)}=\left[\mathbf{X}_{t(\alpha(j))}^{\prime}\right]_{U(\alpha(j)) K(\alpha(j))}$} & $J(j)=K(\alpha(j)), V(j)=U(\alpha(j))$ \\
\hline
\end{tabular}

where

$$
\begin{aligned}
\Pi_{A}(\gamma) & :=\sum_{I, U, V} \chi_{A}(\gamma, U, V) \prod_{i=1}^{n}\left[A_{t(i)}^{\prime}\right]_{I(i), U(i)}\left[A_{t(i)}\right]_{V(i), I(\rho(i))}, \\
\Pi_{B}(\gamma) & :=\sum_{K, J} \chi_{B}(\gamma, K, J) \prod_{i=1}^{n}\left[B_{t(i)}\right]_{K(i), J(i)} .
\end{aligned}
$$

It remains to prove the following two results.

Lemma 3.2. If $\gamma \in \mathcal{F}_{n}(t)$ then

$$
\Pi_{A}(\gamma)=p_{\sigma \odot \gamma, t(\sigma, \gamma)}\left(\Sigma_{1}, \ldots, \Sigma_{s}\right) .
$$

Lemma 3.3. If $\gamma \in \mathcal{F}_{n}(t)$ then $\Pi_{B}(\gamma)=q_{\gamma, t}\left(B_{1}, \ldots, B_{s}\right)$.

Proof of Lemma 3.3 .

Given $K, J:\{1, \ldots, n\} \rightarrow\{1, \ldots, M\}$, define $\widetilde{K}:\{ \pm 1, \ldots, \pm n\} \rightarrow\{1, \ldots, M\}$ by

$$
\widetilde{K}(j)= \begin{cases}K(j) & \text { if } j>0 \\ J(-j) & \text { if } j<0\end{cases}
$$

It is clear that $(K, J) \mapsto \widetilde{K}$ is a bijection between the set of all pairs of functions $K, J:\{1, \ldots, n\} \rightarrow\{1, \ldots, M\}$ and all functions $\widetilde{K}:\{ \pm 1, \ldots, \pm n\} \rightarrow\{1, \ldots, M\}$.

From Table 2 it follows that

$$
\chi_{B}(\gamma, J, K)=\prod_{\{u, v\} \in \gamma} 1_{\widetilde{K}(u)=\widetilde{K}(v)}
$$

For a fixed $\gamma \in \mathcal{F}_{n}$, denote $\alpha=\pi(\gamma),\left(\varepsilon_{j}\right)=\varepsilon(\gamma)$. Then we can factor $\Pi_{B}(\gamma)$ over the cycles of $\alpha$,

$$
\Pi_{B}(\gamma)=\sum_{K, J} \chi_{B}(\gamma, K, J) \prod_{i=1}^{n}\left[B_{t(i)}\right]_{K(i), J(i)}=\prod_{c \in C(\gamma)} \sum_{\left.K\right|_{c},\left.J\right|_{c}} \chi_{B}\left(\gamma,\left.K\right|_{c},\left.J\right|_{c}\right) \prod_{j \in c}\left[B_{t(j)}\right]_{K(j), J(j)}
$$

On a single cycle $c=\left(j_{1}, \ldots, j_{r}\right)$ of $\alpha, t:=\left.t\right|_{c}$ is constant. So to end the proof, it suffices to show that

$$
\sum_{K, J: c \rightarrow\{1, \ldots, M\}} \chi_{B}(\gamma, K, J) \prod_{j \in c}\left[B_{t}\right]_{K(j), J(j)}=\operatorname{tr}\left(\prod_{j \in c} B_{t}^{-\varepsilon_{j}}\right) .
$$

We note that cycle $c$ corresponds to the sequence

$$
C=\left(j_{1}, \pm j_{2}\right),\left(\mp j_{2}, \pm j_{3}\right), \ldots,\left(\mp j_{r-1}, \pm j_{r}\right),\left(\mp j_{r},-j_{1}\right)
$$


of the ordered edges of $\gamma$. The corresponding edges of $\delta$ are

$$
\left.\delta\right|_{C}=\left(\left(-j_{1}, j_{1}\right),\left( \pm j_{2}, \mp j_{2}\right), \ldots,\left( \pm j_{r}, \mp j_{r}\right)\right) .
$$

These two sequences can be written directly in terms of $\left(\varepsilon_{j}\right)$ as

$$
\begin{gathered}
\left.\delta\right|_{C}=\left(-j_{1}, j_{1}\right),\left(-\varepsilon_{j_{2}} j_{2}, \varepsilon_{j_{2}} j_{2}\right), \ldots,\left(-\varepsilon_{j_{r}} j_{r}, \varepsilon_{j_{r}} j_{r}\right) \\
C=\left(j_{1},-\varepsilon_{j_{2}} j_{2}\right),\left(\varepsilon_{j_{2}} j_{2},-\varepsilon_{j_{3}} j_{3}\right), \ldots,\left(\varepsilon_{j_{r-1}} j_{r-1},-\varepsilon_{j_{r}} j_{r}\right),\left(\varepsilon_{j_{r}} j_{r},-j_{1}\right) .
\end{gathered}
$$

For fixed $K, J$, the expression under the sum on the left hand side of (3.7) can be written as

$$
\begin{aligned}
& \chi_{B}(\gamma, K, J) \prod_{j \in c}\left[B_{t}\right]_{K(j), J(j)}=\prod_{i=1}^{r} 1_{\widetilde{K}\left(\varepsilon_{j_{i}} j_{i}\right)=\widetilde{K}\left(-\varepsilon_{j_{i+1}} j_{i+1}\right)}\left[B_{t}\right]_{\widetilde{K}\left(j_{i}\right), \widetilde{K}\left(-j_{i}\right)} \\
& =\prod_{i=1}^{r} 1_{\widetilde{K}\left(\varepsilon_{j_{i}} j_{i}\right)=\widetilde{K}\left(-\varepsilon_{j_{i+1}} j_{i+1}\right)}\left[B_{t}^{-\varepsilon\left(j_{i}\right)}\right]_{\widetilde{K}\left(-\varepsilon_{j_{i}} j_{i}\right), \widetilde{K}\left(\varepsilon_{j_{i}} j_{i}\right)} \\
& =\prod_{i=1}^{r} 1_{\widetilde{K}\left(\varepsilon_{j_{i}} j_{i}\right)=\widetilde{K}\left(-\varepsilon_{j_{i+1}} j_{i+1}\right)}\left[B_{t}^{-\varepsilon\left(j_{i}\right)}\right]_{\widetilde{K}\left(-\varepsilon_{j_{i}} j_{i}\right), \widetilde{K}\left(-\varepsilon_{j_{i+1}} j_{i+1}\right)} .
\end{aligned}
$$

(Here we use cyclic convention $j_{r+1}:=j_{1}$.) Thus the left hand side of (3.7) is

$$
\begin{aligned}
\sum_{\widetilde{K}:\left\{ \pm j_{1}, \ldots, \pm j_{r}\right\} \rightarrow\{1, \ldots, M\}} & \prod_{i=1}^{r} 1_{\widetilde{K}\left(\varepsilon_{j_{i}} j_{i}\right)=\widetilde{K}\left(-\varepsilon_{j_{i+1}} j_{i+1}\right)}\left[B_{t}^{-\varepsilon\left(j_{i}\right)}\right]_{\widetilde{K}\left(-\varepsilon_{j_{i}} j_{i}\right), \widetilde{K}\left(-\varepsilon_{j_{i+1}} j_{i+1}\right)} \\
= & \sum_{\widetilde{K}:\left\{-\varepsilon_{j_{1}} j_{1}, \ldots,-\varepsilon_{j_{r}} j_{r}\right\} \rightarrow\{1, \ldots, M\}} \prod_{i=1}^{r}\left[B_{t}^{-\varepsilon\left(j_{i}\right)}\right]_{\widetilde{K}\left(-\varepsilon_{j_{i}} j_{i}\right), \widetilde{K}\left(-\varepsilon_{j_{i+1}} j_{i+1}\right)}=\operatorname{tr}\left(\prod_{j \in c} B_{t}^{-\varepsilon_{j}}\right),
\end{aligned}
$$

proving (3.7).

\section{Proof of Lemma 3.2.}

We begin by rewriting $\Pi_{A}(\gamma)$ in terms of matrices $\Sigma_{1}, \ldots, \Sigma_{s}$. Recall that $\rho=\pi(\sigma) \in \mathcal{S}_{n}$. We claim that with $\widetilde{I}:\{ \pm 1, \ldots, \pm n\} \rightarrow\{1, \ldots, N\}$ defined by

$$
\widetilde{I}(j):= \begin{cases}I(j) & \text { if } j>0 \\ I(\rho(-j)) & \text { if } j<0\end{cases}
$$

and with $t:\{ \pm 1, \ldots, \pm n\} \rightarrow\{1, \ldots, s\}$ extended by $t(u):=t(|u|)$, we have

$$
\Pi_{A}(\gamma)=\sum_{I:\{1, \ldots, n\} \rightarrow\{1, \ldots, N\}} \prod_{\{u, v\} \in \gamma}\left[\Sigma_{t(u)}\right]_{\widetilde{I}(u), \widetilde{I}(v)} .
$$

To prove (3.11), we re-parameterize each pair of functions $U, V$ by a single function $\widetilde{U}:\{ \pm 1, \ldots, \pm n\} \rightarrow$ $\{1, \ldots, N\}$ defined by

$$
\widetilde{U}(j):= \begin{cases}U(j) & \text { if } j>0 \\ V(-j) & \text { if } j<0\end{cases}
$$

By inspecting the rows of Table 2 we see that

$$
\chi_{A}(\gamma, U, V)=\prod_{\{u, v\} \in \gamma} 1_{\widetilde{U}(u)=\widetilde{U}(v)} .
$$

For fixed $I$,

$$
\sum_{U, V} \chi_{A}(\gamma, U, V) \prod_{i=1}^{n}\left[A_{t(i)}^{\prime}\right]_{I(i), U(i)}\left[A_{t(i)}\right]_{V(i), I(\rho(i))}
$$


factors over the cycles of $\alpha$ into the product of

$$
\sum_{U, V: c \rightarrow\{1, \ldots, N\}} \chi_{A}(\gamma, U, V) \prod_{j \in c}\left[A_{t(j)}^{\prime}\right]_{I(j), U(j)}\left[A_{t(j)}\right]_{V(j), I(\rho(j))} .
$$

The corresponding set $C$ of ordered edges of $\gamma$ is

$$
C=\left(u_{1}, v_{1}\right),\left(u_{2}, v_{2}\right), \ldots,\left(u_{r}, v_{r}\right)
$$

and, as in the proof of Lemma 3.3. we have $u_{k+1}=-v_{k}, k=1, \ldots, r$ with $u_{r+1}:=u_{1}>0$. Of course, $c=\left(j_{1}, \ldots, j_{r}\right)=\left(\left|u_{1}\right|, \ldots,\left|u_{r}\right|\right)$, and $t$ is constant on $c$. Thus, using the symmetry of $A$,

$$
\begin{aligned}
\prod_{j \in c}\left[A_{t(j)}^{\prime}\right]_{I(j), U(j)}\left[A_{t(j)}\right]_{V(j), I(\rho(j))}=\prod_{k=1}^{r}\left[A_{t}\right]_{\widetilde{U}\left(j_{k}\right), \widetilde{I}\left(j_{k}\right)}\left[A_{t}\right]_{\widetilde{U}\left(-j_{k}\right), \widetilde{I}\left(-j_{k}\right)} & =\prod_{k=1}^{r}\left[A_{t}\right]_{\widetilde{I}\left(-v_{k}\right), \widetilde{U}\left(-v_{k}\right)}\left[A_{t}\right]_{\widetilde{U}\left(v_{k+1}\right), \widetilde{I}\left(v_{k+1}\right)} .
\end{aligned}
$$

Since $\chi_{A}=1$ implies $\widetilde{U}\left(u_{k}\right)=\widetilde{U}\left(v_{k}\right)$, we see that

$$
\widetilde{U}\left(v_{k+1}\right)=\widetilde{U}\left(-v_{k}\right) .
$$

Thus,

$$
\begin{aligned}
& \sum_{U, V: c \rightarrow\{1, \ldots, N\}} \chi_{A}(\gamma, U, V) \prod_{j \in c}\left[A_{t(j)}^{\prime}\right]_{I(j), U(j)}\left[A_{t(j)}\right]_{V(j), I(\rho(j))} \\
&= \sum_{\widetilde{U}:\left\{ \pm j_{1}, \ldots, \pm j_{r}\right\} \rightarrow\{1, \ldots, N\}} \prod_{k=1}^{r} 1_{\widetilde{U}\left(v_{k+1}\right)=\widetilde{U}\left(-v_{k}\right)}\left[A_{t}\right]_{\widetilde{I}\left(-v_{k}\right), \widetilde{U}\left(-v_{k}\right)}\left[A_{t}\right]_{\widetilde{U}\left(v_{k+1}\right), \widetilde{I}\left(v_{k+1}\right)} \\
&=\sum_{\widetilde{U}:\left\{-v_{1}, \ldots,-v_{r}\right\} \rightarrow\{1, \ldots, N\}}\left[A_{t}\right]_{\widetilde{I}\left(-v_{k}\right), \widetilde{U}\left(-v_{k}\right)}\left[A_{t}\right]_{\widetilde{U}\left(-v_{k}\right), \widetilde{I}\left(v_{k+1}\right)}=\prod_{\{u, v\} \in C}\left[\Sigma_{t}\right]_{\widetilde{I}(u), \widetilde{I}(v)} .
\end{aligned}
$$

We now fix $\gamma \in \mathcal{F}_{n}(t)$ and consider (3.11), which we want to factor over the cycles of $\beta=\pi(\sigma \odot \gamma)$. To do so, let $\tau$ be the bijection from pairs $\{u, v\} \in \gamma$ onto the corresponding pairs $\{r, s\} \in \sigma \odot \gamma$ which appeared in Definition 2.8 see Table 3 .

Since $\tau$ is a bijection and the cycles of $\beta$ are in one-to-one correspondence with the sequences of pairs in $\sigma \odot \gamma$, to prove (3.4) it suffices to show that if $c$ is a cycle of $\beta$ and $C$ is the corresponding sequence of pairs in $\sigma \odot \gamma$, then

$$
\prod_{\{u, v\} \in \tau^{-1}(C)}\left[\Sigma_{t(u)}\right]_{\widetilde{I}(u), \widetilde{I}(v)}=\prod_{j \in c}\left[\Sigma_{t(\sigma, \gamma)(j)}\right]_{I(j), I \circ \beta(j)}
$$

We prove (3.15) by showing that each of the factors on the left hand side of (3.15) can be rewritten into the corresponding factor on the right hand side of (3.15). To do so, let $\{u, v\}=\tau^{-1}(\{r, s\})$, where $\{r, s\} \in C$ are such that $|r|,|s|$ are the consecutive numbers in cycle $c$, i.e., $|s|=\beta(|r|)$.

We note that $\tau$ preserves the number of positive elements in each pair $\{u, v\}$; thus if $u, v>0$ then $r, s>0$ and similarly if $u v<0$ then $r s<0$; Table 3 lists all possible cases with the intermediate three-row diagram.

We now consider all possible cases.

A-1 Suppose $r<0, s>0$. Since $\Sigma_{t(u)}=\Sigma_{t(v)}$ is symmetric, without loss of generality we may assume $u>0, v<0$. Then $u=s$ and $r=-\rho(-v)$, so (3.10) gives

$$
\left[\Sigma_{t(u)}\right]_{\widetilde{I}(u), \widetilde{I}(v)}=\left[\Sigma_{t(-v)}\right]_{\widetilde{I}(v), \widetilde{I}(u)}=\left[\Sigma_{t \circ \rho^{-1}(|r|)}\right]_{I(|r|), I(s)}=\left[\Sigma_{t(\sigma, \gamma)(|r|)}\right]_{I(|r|), I \circ \beta(|r|)} .
$$

A-2 Suppose $r>0, s<0$. Since $\Sigma_{t(u)}=\Sigma_{t(v)}$ is symmetric, without loss of generality we may assume $u>0, v<0$. Then $u=r$ and $s=-\rho(-v)$, so (3.10) gives

$$
\left[\Sigma_{t(u)}\right]_{\widetilde{I}(u), \widetilde{I}(v)}=\left[\Sigma_{t(|r|)}\right]_{I(r), I(|s|)}=\left[\Sigma_{t(\sigma, \gamma)(|r|)}\right]_{I(|r|), I \circ \beta(|r|)} .
$$


Table 3: Bijection $\tau$ from pairs $\{u, v\} \in \gamma$ to pairs $\{r, s\} \in \sigma \odot \gamma$. Here $\rho=\pi(\sigma) \in \mathcal{S}_{n}$.

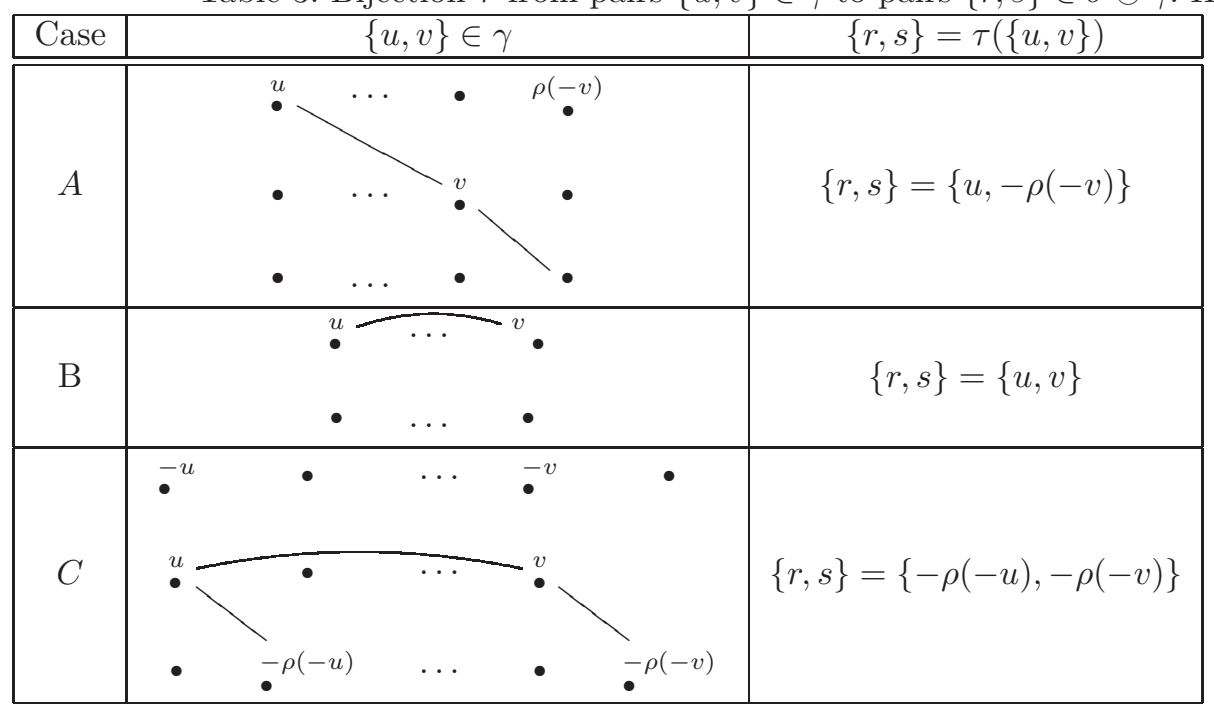

B Suppose $r>0, s>0$. Then $\{u, v\}=\{r, s\}$ and (3.10) gives

$$
\left[\Sigma_{t(u)}\right]_{\widetilde{I}(u), \widetilde{I}(v)}=\left[\Sigma_{t(r)}\right]_{I(r), I(s)}=\left[\Sigma_{t(|r|)}\right]_{I(|r|), I \circ \beta(|r|)},
$$

C Suppose $r<0, s<0$. Then swapping the roles of $u, v$ if necessary, we may assume that $r=-\rho(-u)$ and $s=-\rho(-v)$, so (3.10) gives

$$
\left[\Sigma_{t(u)}\right]_{\widetilde{I}(u), \widetilde{I}(v)}=\left[\Sigma_{t \circ \rho^{-1}(|r|)}\right]_{I(|r|), I(|s|)}=\left[\Sigma_{t(\sigma, \gamma)(|r|)}\right]_{I(|r|), I \circ \beta(|r|)} .
$$

Proof of Theorem 2.18. This proof is similar to the proof of Theorem 2.9 with (1.2) used instead of Wick formula. Throughout the proof, we denote $\rho=\pi(\sigma), \beta=\pi(\sigma \odot \gamma)$. For functions $I:\{1, \ldots, n\} \rightarrow\{1, \ldots, N\}$ and $J:\{1, \ldots, n\} \rightarrow\{1, \ldots, M\}$, we denote by $\widetilde{I}, \widetilde{J}$ functions on $\{ \pm 1, \ldots, \pm n\}$, which are defined by (3.10) and $\widetilde{J}(j):=J(|j|)$, respectively. We also extend $t$ to $\{ \pm 1, \ldots, \pm n\}$ by $t(s):=t(|s|)$; we will use the same symbol $t$ for this extension. From (2.2) and (1.3) we see that

$$
\tau\left(p_{\sigma, t}\left(\mathbf{W}_{1}, \ldots, \mathbf{W}_{s}\right)\right)=\sum_{I, J} \tau\left(\left[\mathbf{X}_{t(1)}\right]_{\widetilde{J}(1), \widetilde{I}(1)}\left[\mathbf{X}_{t(1)}\right]_{\widetilde{J}(-1), \widetilde{I}(-1)} \ldots\left[\mathbf{X}_{t(n)}\right]_{\widetilde{J}(n), \widetilde{I}(n)}\left[\mathbf{X}_{t(n)}\right]_{\widetilde{J}(-n), \widetilde{I}(-n)}\right)
$$

By (1.2), this implies

$$
\tau\left(p_{\sigma, t}\left(\mathbf{W}_{1}, \ldots, \mathbf{W}_{s}\right)\right)=\sum_{I, J} \sum_{\gamma \in \mathcal{F}_{n}} q^{\operatorname{cr}(\gamma)} \prod_{\{u, v\} \in \gamma} \tau\left(\left[\mathbf{X}_{t(u)}\right]_{\widetilde{J}(u), \widetilde{I}(u)}\left[\mathbf{X}_{t(v)}\right]_{\widetilde{J}(v), \widetilde{I}(v)}\right) .
$$

Since $\tau\left(\left[\mathbf{X}_{t(u)}\right]_{\widetilde{J}(u), \widetilde{I}(u)}\left[\mathbf{X}_{t(v)}\right]_{\widetilde{J}(v), \widetilde{I}(v)}\right)=0$ when $t(u) \neq t(v)$, we see that the sum can be restricted to the color-preserving partitions $\gamma \in \mathcal{F}_{n}(t)$. Changing the order of summation, and taking into account (1.2), we get

$$
\tau\left(p_{\sigma, t}\left(\mathbf{W}_{1}, \ldots, \mathbf{W}_{s}\right)\right)=\sum_{\gamma \in \mathcal{F}_{n}(t)} q^{\operatorname{cr}(\gamma)} \sum_{I, J} \prod_{\{u, v\} \in \gamma} \tau\left(\left[\mathbf{X}_{t(u)}\right]_{\widetilde{J}(u), \widetilde{I}(u)}\left[\mathbf{X}_{t(v)}\right]_{\widetilde{J}(v), \widetilde{I}(v)}\right)=\sum_{\gamma \in \mathcal{F}_{n}(t)} q^{\operatorname{cr}(\gamma)} \Pi_{1}(\gamma) \Pi_{2}(\gamma)
$$

where

$$
\begin{aligned}
& \Pi_{1}(\gamma)=\sum_{J:\{1, \ldots, n\} \rightarrow\{1, \ldots, M\}} \prod_{\{u, v\} \in \gamma}\left[B_{t(u)}\right]_{\widetilde{J}(u), \widetilde{J}(v)}, \\
& \Pi_{2}(\gamma)=\sum_{I:\{1, \ldots, n\} \rightarrow\{1, \ldots, N\}} \prod_{\{u, v\} \in \gamma}\left[\Sigma_{t(u)}\right]_{\widetilde{I}(u), \widetilde{I}(v)} .
\end{aligned}
$$

To end the proof, it remains to show that

$$
\Pi_{1}(\gamma)=p_{\gamma, t}\left(B_{1}, \ldots, B_{s}\right)
$$

and

$$
\Pi_{2}(\gamma)=p_{\sigma \odot \gamma, t(\sigma, \gamma)}\left(\Sigma_{1}, \ldots, \Sigma_{s}\right)
$$

The proof of (3.18) is similar to the proof of Lemma 3.3 and is omitted. Since the right hand side of (3.17) is the same as the right hand side of (3.11), formula (3.19) follows from the proof of Lemma 3.2 


\section{Proof of Theorem 1.7}

The identification of the limit in Theorem 1.7 when $q=0$ or $q=1$ is a consequence of the following general property of $q$-Wishart matrices.

Theorem 4.1. Let

$$
\mathbf{W}_{1}^{(N)}, \mathbf{W}_{2}^{(N)}, \ldots, \mathbf{W}_{s}^{(N)}, \widetilde{\mathbf{W}}_{1}^{(N)}, \widetilde{\mathbf{W}}_{2}^{(N)}, \ldots, \widetilde{\mathbf{W}}_{s}^{(N)} \in \mathcal{W}_{q}\left(\frac{1}{N} I_{N}, I_{M}\right)
$$

be q-orthogonal with $M=M(N)$ such that $M / N \rightarrow \lambda$. Fix a polynomial $Q\left(x_{1}, \ldots, x_{s}\right)$ in noncommutative variables and let

$$
X_{N}=\operatorname{tr}\left(Q\left(\mathbf{W}_{1}^{(N)}, \ldots, \mathbf{W}_{s}^{(N)}\right)\right)-\tau\left(\operatorname{tr}\left(Q\left(\mathbf{W}_{1}^{(N)}, \ldots, \mathbf{W}_{s}^{(N)}\right)\right)\right)
$$

and

$$
Y_{N}=\operatorname{tr}\left(Q\left(\widetilde{\mathbf{W}}_{1}^{(N)}, \ldots, \widetilde{\mathbf{W}}_{s}^{(N)}\right)\right)-\tau\left(\operatorname{tr}\left(Q\left(\widetilde{\mathbf{W}}_{1}^{(N)}, \ldots, \widetilde{\mathbf{W}}_{s}^{(N)}\right)\right)\right) .
$$

Then for $m \geq 0$ the limits $\lim _{N \rightarrow \infty} \tau\left(X_{N}^{2}\right), \lim _{N \rightarrow \infty} \tau\left(\left(X_{N}+Y_{N}\right)^{m}\right)$ exist, and

$$
\lim _{N \rightarrow \infty}\left(\tau\left(\left(X_{N}-Y_{N}\right)^{2}\left(X_{N}+Y_{N}\right)^{m}\right)-2 \tau\left(X_{N}^{2}\right) \tau\left(\left(X_{N}+Y_{N}\right)^{m}\right)\right)=0 .
$$

Remark 4.2. Since the conditional expectation of $X_{N}$ given $X_{N}+Y_{N}$ is $\left(X_{N}+Y_{N}\right) / 2$, expression (4.1) can be interpreted as the statement that the conditional variance of $X_{N}$ given $X_{N}+Y_{N}$ is asymptotically constant. In the self-adjoint case, the latter property identifies the distribution when $q=0,1$, see [5, Theorem 3.1]. We note that Theorem 4.1 holds true for all $-1 \leq q \leq 1$, but Examples 4.6 4.8 and 4.7 show the limiting law may fail to be $q$-Gaussian for $0<|q|<1$. This suggests that there is no natural $q$-version of $[5$.

\subsection{Auxiliary combinatorial result}

The proof of Theorem 4.1 relies on the following partition version of a theorem of Jaques 22 .

Proposition 4.3. If $\gamma \in \mathcal{F}_{n}$ and $\sigma \in \mathcal{F}_{n}^{+}$are such that the graph $\gamma \cup \sigma \cup \delta$ is connected, then there exists an integer $h \geq 0$ such that

$$
\# \mathcal{C}(\gamma)+\# \mathcal{C}(\sigma)+\# \mathcal{C}(\sigma \odot \gamma)-n=2-h .
$$

For the proof, we will need to consider $\mathcal{F}_{n}$ in all three roles: as pair partitions, as graphs, and as a subset of $\mathcal{S}_{2 n}$. To help distinguish between these interpretations, denote by $z(\alpha)$ the number of cycles of a permutation $\alpha$. Recall that in this notation, if $\gamma \in \mathcal{F}_{n}$ then $\# \mathcal{C}(\gamma)=z(\pi(\gamma))$. We will use the above mentioned result of Jaques, which we now state in the version most convenient for our purposes.

Theorem A. 220 If $\alpha, \beta \in \mathcal{S}_{n}$ act transitively on $\{1, \ldots, n\}$, then there exists an integer $g \geq 0$ such that

$$
z(\alpha)+z(\beta)+z\left(\beta^{-1} \alpha\right)-n=2-2 g .
$$

(For an accessible algebraic proof see [29, Theorem 1]. For a graph-theoretic proof, see [27, Proposition 1.5.3].)

Proof of Proposition 4.3. The proof consists of two claims, and an application of (4.3). As noted in the proof of [13, Lemma 3.5],

Claim 1. For $\gamma \in \mathcal{F}_{n}$,

$$
z(\gamma \circ \delta)=2 \# \mathcal{C}(\gamma)
$$

(This is because every cycle of $\delta \cup \gamma$ splits into two cycles of $\gamma \circ \delta$. Recall that $\delta$ is defined by (2.1).)

We now note the following.

Claim 2. If $\gamma \in \mathcal{F}_{n}, \sigma \in \mathcal{F}_{n}^{+}$and the graph $\gamma \cup \sigma \cup \delta$ is connected, then the group generated by permutations $\gamma \circ \delta, \sigma \circ \delta$ has at most two orbits on $\{ \pm 1, \ldots, \pm n\}$.

To prove this claim, we note that for $\sigma \in \mathcal{F}_{n}^{+}$the cycles $\sigma \circ \delta$ are exactly the cycles $c_{1}, \ldots, c_{k}$ of $\pi(\sigma) \in \mathcal{S}_{n}$ and $-c_{1}, \ldots,-c_{k}$.

Since the graph $\gamma \cup \sigma \cup \delta$ is connected, $\gamma$ must connect the sets

$$
c_{1} \cup-c_{1}, c_{2} \cup-c_{2}, \ldots, c_{k} \cup-c_{k},
$$


so $\gamma \circ \delta$ must also connect these sets. Thus if $S$ is an orbit of $\langle\sigma \circ \delta, \gamma \circ \delta\rangle$, then $\{|j|: j \in S\}=\{1, \ldots, n\}$.

As the mapping $S \ni j \mapsto|j| \in\{1, \ldots, n\}$ is two-valued and onto, there cannot be more than two disjoint inverse images of $\{1, \ldots, n\}$.

By Claim 2, applying (4.3) to $\alpha=\gamma \circ \delta, \beta=\sigma \circ \delta \in \mathcal{S}_{2 n}$ or to their restrictions on the two orbits separately, we have

$$
z(\gamma \circ \delta)+z(\sigma \circ \delta)+z\left((\sigma \circ \delta)^{-1} \circ \gamma \circ \delta\right)-2 n= \begin{cases}2-2 g & \text { if }\langle\sigma \circ \delta, \gamma \circ \delta\rangle \text { acts transitively } \\ 4-2 g_{1}-2 g_{2} & \text { otherwise }\end{cases}
$$

for some integers $g, g_{1}, g_{2} \geq 0$. Thus in both cases, there is an integer $h \geq 0$ such that

$$
z(\gamma \circ \delta)+z(\sigma \circ \delta)+z\left((\sigma \circ \delta)^{-1} \circ \gamma \circ \delta\right)-2 n=4-2 h .
$$

We now note that $(\sigma \circ \delta)^{-1}=\hat{\sigma} \circ \delta$, where $\hat{\sigma}(j)=-\sigma(-j)$. Moreover, $\hat{\sigma} \circ \delta=\delta \circ \hat{\sigma}$ so permutation $=(\sigma \circ \delta)^{-1} \circ$ $\gamma \circ \delta=\hat{\sigma} \circ \delta \circ \gamma \circ \delta$ is similar to $\hat{\sigma} \circ \gamma$.

In the proof of Corollary 2.17 we already noted that $\# \mathcal{C}(\sigma \odot \gamma)$ is the number of components of the graph $\hat{\sigma} \cup \gamma$. We now observe that the cycles of $\hat{\sigma} \circ \gamma$ split each such component in two. Therefore

$$
z\left((\sigma \circ \delta)^{-1} \circ \gamma \circ \delta\right)=z(\hat{\sigma} \circ \gamma)=2 \# \mathcal{C}(\sigma \circ \gamma)
$$

This, together with Claim 1 and (4.5) gives (4.2).

\subsection{Proofs of Theorems 4.1 and 1.7}

Our first goal is to show that the limits of joint moments exist; the main step consists of analyzing moments of the monomials.

We will need additional notation. By $\mathcal{F}_{n}(t, \sigma)$ we denote the set of all $\gamma \in \mathcal{F}_{n}(t)$ which connect every cycle of $\sigma$ to some other cycle. That is, all components of the graph $\delta \cup \sigma \cup \gamma$ are strictly larger than the components of $\sigma \cup \delta$.

Lemma 4.4. Let $\mathbf{W}_{1}, \mathbf{W}_{2}, \ldots, \mathbf{W}_{s} \in \mathcal{W}_{q}\left(\frac{1}{N} I_{N}, I_{M}\right)$ be q-orthogonal. Suppose that the cycles $c_{u}(1 \leq u \leq r)$ of $\sigma \in \mathcal{F}_{n}^{+}$consist of consecutive integers, as in (2.2). Fix $t:\{1, \ldots, n\} \rightarrow\{1, \ldots, s\}$. Then

$$
\tau\left(\prod_{u=1}^{r}\left(\operatorname{tr}\left(\prod_{j \in c_{u}} \mathbf{W}_{t(j)}\right)-\tau\left(\operatorname{tr}\left(\prod_{j \in c_{u}} \mathbf{W}_{t(j)}\right)\right)\right)=\sum_{\gamma \in \mathcal{F}_{n}(t, \sigma)} q^{\operatorname{cr}(\gamma)} M^{\# \mathcal{C}(\gamma)} N^{\# \mathcal{C}(\sigma \odot \gamma)-n} .\right.
$$

Proof. Under the standard convention that empty product is identity, the left hand side of (4.6) is

$$
\sum_{F \subset\{1, \ldots, r\}}(-1)^{\# F} \tau\left(\prod_{u \notin F} \operatorname{tr}\left(\prod_{j \in c_{u}} \mathbf{W}_{t(j)}\right)\right) \prod_{u \in F} \tau\left(\operatorname{tr}\left(\prod_{j \in c_{u}} \mathbf{W}_{t(j)}\right)\right) .
$$

(Here, finite subsets $F \subset\{1, \ldots, r\}$ and their complements in $\{1, \ldots, r\}$ are treated as ordered sets with the natural order on integers, so that the noncommutative products of traces that occur in the first factor are well defined. That is, if $F=\left\{u_{1}, u_{2}, \ldots, u_{m}\right\}$ with $u_{1}<u_{2}<\cdots<u_{m}$ then $\prod_{u \in F} x_{u}:=x_{u_{1}} x_{u_{2}} \ldots x_{u_{m}}$ is well defined.)

We now re-rewrite the right hand side of (4.6). Let $c_{1}, \ldots, c_{r}$ be the cycles of $\sigma$ in increasing order, and let $k_{1}, \ldots, k_{r}$ denote their lengths. (Recall our convention that $c_{1}, \ldots, c_{r}$ are in fact the cycles of $\pi(\sigma) \in \mathcal{S}_{n}$.)

For $1 \leq u \leq r$, let $\mathcal{G}_{u}$ denote the set of $\gamma \in \mathcal{F}_{n}(t)$ which "isolate" cycle $c_{u}$, i.e., do not connect cycle $c_{u}$ to any other cycle. Then $\mathcal{F}_{n}(t, \sigma)=\mathcal{F}_{n}(t) \backslash \bigcup_{u=1}^{r} \mathcal{G}_{u}$, so for any weighted counting measure $H$,

$$
H\left(\mathcal{F}_{n}(t, \sigma)\right)=H\left(\mathcal{F}_{n}(t)\right)-H\left(\bigcup_{u=1}^{r} \mathcal{G}_{u}\right)=\sum_{F \subset\{1, \ldots, r\}}(-1)^{\# F} H\left(\bigcap_{u \in F} \mathcal{G}_{u}\right)
$$

where $H\left(\mathcal{F}_{n}(t)\right)$ corresponds to $F=\emptyset$ in the expansion. Therefore, the right hand side of (4.6) is

$$
\sum_{F \subset\{1, \ldots, r\}}(-1)^{\# F} \sum_{\gamma \in \bigcap_{u \in F} \mathcal{G}_{u}} q^{\operatorname{cr}(\gamma)} M^{\# \mathcal{C}(\gamma)} N^{\# \mathcal{C}(\sigma \odot \gamma)-n} .
$$


Note that $\gamma \in \bigcap_{u \in F} \mathcal{G}_{u}$ can be uniquely split into the disjoint union $\gamma_{0} \cup \bigcup_{u \in F} \gamma_{u}$, where $\gamma_{u} \in \mathcal{F}_{k_{u}}$ is a pair partition of cycle $c_{u}$, and $\gamma_{0} \in \mathcal{F}_{n_{0}}$ with $n_{0}=n-\sum_{u \in F} k_{u}$. We now note that $\sigma \odot \gamma=\left(\sigma_{0} \odot \gamma_{0}\right) \cup \bigcup_{u \in F} c_{u} \odot \gamma_{u}$. Since the cycles of $\sigma$ consist of consecutive segments, no additional crossings of $\gamma$ can arise from the edges connecting $c_{u}$ besides the crossings already accounted for as crossings of $\gamma_{u}$. Thus $\operatorname{cr}(\gamma)=\operatorname{cr}\left(\gamma_{0}\right)+\sum_{u \in F} \operatorname{cr}\left(\gamma_{u}\right)$, and the right hand side of (4.6) can be written as

$$
\sum_{F \subset\{1, \ldots, r\}}(-1)^{\# F} \sum_{\gamma_{0} \in \mathcal{F}_{n_{0}}} q^{\operatorname{cr}\left(\gamma_{0}\right)} M^{\# \mathcal{C}\left(\gamma_{0}\right)} N^{\# \mathcal{C}\left(\sigma_{0} \odot \gamma_{0}\right)-n_{0}} \prod_{u \in F} \sum_{\gamma_{u} \in \mathcal{F}_{k_{u}}} q^{\mathrm{cr}\left(\gamma_{u}\right)} M^{\# \mathcal{C}\left(\gamma_{u}\right)} N^{\# \mathcal{C}\left(c_{u} \odot \gamma_{u}\right)-k_{u}}
$$

Applying (2.14) to each of the factors, we arrive at (4.7), thus deriving the left hand side of (4.6).

Next, we show that the non-negligible terms in expansion (4.6) correspond to $\gamma$ that connect pairs of cycles of $\sigma$.

Lemma 4.5. Fix $\gamma \in \mathcal{F}_{n}(t, \sigma)$. Then the limit

$$
M_{n}(\gamma):=\lim _{N \rightarrow \infty, M / N \rightarrow \lambda} M^{\# \mathcal{C}(\gamma)} N^{\# \mathcal{C}(\sigma \odot \gamma)-n}
$$

exists. Moreover, if the number of cycles of $\sigma$ connected by $\gamma$ is more than 2, then $M_{n}(\gamma)=0$. If $\gamma$ connects only pairs $c_{u}, c_{v}$ of cycles of $\sigma$, and $\gamma_{u, v}$ denotes the corresponding part of $\gamma$, then

$$
M_{n}(\gamma)=\prod_{\{u, v\}} M_{2}\left(\gamma_{u, v}\right)
$$

(The product is taken over all unordered pairs $u, v$ corresponding to the connected cycles of $\sigma$ ).

Proof. Since $\gamma \in \mathcal{F}_{n}(t, \sigma)$, once we decompose $\sigma \cup \gamma \cup \delta$ into connected components, the decomposition splits the cycles of $\sigma$ into groups of $m_{1}, \ldots, m_{r} \geq 2$ cycles, perhaps non-consecutive. This partitioning splits $\gamma, \sigma$ and $\sigma \odot \gamma$ into disjoint triplets $\gamma_{u}, \sigma_{u}, \sigma_{u} \odot \gamma_{u} \in \mathcal{F}_{n_{u}}, u=1,2, \ldots, r$, with $n=n_{1}+\cdots+n_{r}$, which contribute multiplicatively,

$$
M^{\# \mathcal{C}(\gamma)} N^{\# \mathcal{C}(\sigma \odot \gamma)-n}=(M / N)^{\# \mathcal{C}(\gamma)} \prod_{u=1}^{r} N^{\# \mathcal{C}\left(\gamma_{u}\right)+\# \mathcal{C}\left(\sigma_{u} \odot \gamma_{u}\right)+\# \mathcal{C}\left(\sigma_{u}\right)-n_{u}-m_{u}}
$$

(Recall that $m_{u}=\# \mathcal{C}\left(\sigma_{u}\right)$.) Since the graphs of $\sigma_{u} \cup \gamma_{u} \cup \delta_{u}$ are connected, by formula (4.2), there are integers $h_{1}, \ldots h_{r} \geq 0$ such that

$$
M^{\# \mathcal{C}(\gamma)} N^{\# \mathcal{C}(\sigma \odot \gamma)-n}=(M / N)^{\# \mathcal{C}(\gamma)} \prod_{u=1}^{r} N^{2-m_{u}-h_{u}} .
$$

Therefore, the limit always exists. The limit is zero if at least one of the $m_{u}>2$ or at least one of the $h_{u}>0$. If all groups are pairs, i.e., $m_{1}=m_{2}=\cdots=m_{r}=2$, then the limit factors into the contributions from the pairs of cycles of $\sigma$; of course, the only $\gamma$ that contribute to this limit are those with $h_{1}=h_{2}=\cdots=h_{r}=0$.

Proof of Theorem 4.1. Since $(x-y)^{2}=x(x-y)+y(y-x)$, and joint moments of $\left(X_{N}, Y_{N}\right)$ are symmetric in $X, Y$, it is enough to show that

$$
\lim _{N \rightarrow \infty}\left(\tau\left(X_{N}\left(X_{N}-Y_{N}\right)\left(X_{N}+Y_{N}\right)^{m}\right)-\tau\left(X_{N}^{2}\right) \tau\left(\left(X_{N}+Y_{N}\right)^{m}\right)\right)=0
$$

After re-indexing the $q$-Wishart matrices so that $\mathbf{W}_{-j}:=\widetilde{\mathbf{W}}_{j}$, we can represent $X_{N}=\sum \alpha_{u} X_{N, u}$ and $Y_{N}=\sum \alpha_{u} Y_{N, u}$ as finite linear combinations (with the same coefficients, and the same choice of functions $\left.t_{u}: c_{u} \rightarrow\{1, \ldots, s\}\right)$ of the centered monomials

$$
\begin{aligned}
X_{N, u} & =\operatorname{tr}\left(\prod_{j \in c_{u}} \mathbf{W}_{t_{u}(j)}\right)-\tau\left(\operatorname{tr}\left(\prod_{j \in c_{u}} \mathbf{W}_{t_{u}(j)}\right)\right) \\
Y_{N, u} & =\operatorname{tr}\left(\prod_{j \in c_{u}} \mathbf{W}_{-t_{u}(j)}\right)-\tau\left(\operatorname{tr}\left(\prod_{j \in c_{u}} \mathbf{W}_{-t_{u}(j)}\right)\right) .
\end{aligned}
$$

(Here $c_{u}$ are finite sets of integers of prescribed cardinality, which will be assembled into the cycles of $\sigma$ in the next step.) 
Expanding (4.9) into the monomials, it is enough to show that

$$
\lim _{N \rightarrow \infty}\left(\tau\left(X_{N, v_{0}}\left(X_{N, u}-Y_{N, u}\right) \prod_{j=1}^{m}\left(X_{N, v_{j}}+Y_{N, v_{j}}\right)\right)-\tau\left(X_{N, v_{0}} X_{N, u}\right) \tau\left(\prod_{j=1}^{m}\left(X_{N, v_{j}}+Y_{N, v_{j}}\right)\right)\right)=0
$$

for all admissible indexes $v_{0}, \ldots, v_{m}, u$. Once $v_{0}, u, v_{1}, \ldots, v_{m}$ are fixed, we modify $t$ and the sets $c_{u}$ so that $c_{v_{0}}, c_{u}, c_{v_{1}}, \ldots, c_{v_{m}}$ are consecutive blocks of integers that form the cycles of $\sigma \in \mathcal{F}_{n}^{+}$. This allows us to apply Lemmas 4.4 and 4.5, which imply that the limits of moments exist. Expanding the products

$$
\prod_{j=1}^{n}\left(X_{N, v_{j}}+Y_{N, v_{j}}\right)=\sum_{\eta_{1}, \ldots, \eta_{n}=0,1} \prod_{j=1}^{m} X_{N, v_{j}}^{\eta_{j}} Y_{N, v_{j}}^{1-\eta_{j}}
$$

we see that it suffices to show that

$$
\begin{aligned}
\lim _{N \rightarrow \infty} \sum_{\eta_{1}, \ldots, \eta_{m}=0,1} \tau\left(X_{N, v_{0}}\left(X_{N, u}-Y_{N, u}\right) \prod_{j=1}^{m} X_{N, v_{j}}^{\eta_{j}} Y_{N, v_{j}}^{1-\eta_{j}}\right) & \\
& =\lim _{N \rightarrow \infty} \sum_{\eta_{1}, \ldots, \eta_{m}=0,1} \tau\left(X_{N, v_{0}} X_{N, u}\right) \tau\left(\prod_{j=1}^{m} X_{N, v_{j}}^{\eta_{j}} Y_{N, v_{j}}^{1-\eta_{j}}\right) .
\end{aligned}
$$

To shorten the notation, denote by $c_{0}, c, c_{1}, \ldots, c_{m}$ the cycles $c_{v_{0}}, c_{u}, c_{v_{1}}, \ldots, c_{v_{m}}$ of $\sigma$. In view of (4.10,4.11] Lemmas 4.4 and 4.5 imply that for each choice of $\eta=\left(\eta_{1}, \ldots, \eta_{m}\right) \in\{0,1\}^{n}$ there is $t_{\eta}:\{1, \ldots,\} \rightarrow\{ \pm 1, \ldots, \pm s\}$ such that

$$
\tau\left(X_{N, v_{0}} X_{N, u} \prod_{j=1}^{m} X_{N, v_{j}}^{\eta_{j}} Y_{N, v_{j}}^{1-\eta_{j}}\right)=\sum_{\gamma \in \mathcal{F}_{n}\left(t_{\eta}, \sigma\right)} q^{\operatorname{cr}(\gamma)} M_{m+2}(\gamma)+O(1 / N) .
$$

For $j=0, \ldots, m$ denote by $\mathcal{H}_{j}\left(t_{\eta}\right)$ the set of $\gamma \in \mathcal{F}_{n}\left(t_{\eta}, \sigma\right)$ which connect cycle $c$ to cycle $c_{j}$ and do not connect the pair $c, c_{j}$ to any other cycles of $\sigma$. Lemma 4.5 implies that (4.15) can be expanded as

$$
\tau\left(X_{N, v_{0}} X_{N, u} \prod_{j=1}^{m} X_{N, v_{j}}^{\eta_{j}} Y_{N, v_{j}}^{1-\eta_{j}}\right)=\sum_{\gamma \in \mathcal{H}_{0}\left(t_{\eta}\right)} q^{\mathrm{cr}(\gamma)} M_{m+2}(\gamma)+\sum_{j=1}^{m} \sum_{\gamma \in \mathcal{H}_{j}\left(t_{\eta}\right)} q^{\operatorname{cr}(\gamma)} M_{m+2}(\gamma)+O(1 / N)
$$

All $\gamma \in \mathcal{H}_{0}\left(t_{\eta}\right)$ can be decomposed into $\gamma_{0} \cup \gamma^{\prime}$, where $\gamma_{0}$ pairs the cycles $c_{0}, c$ only, and $\gamma^{\prime}$ is the pairing of the elements underlying cycles $c_{1}, \ldots, c_{n}$. Furthermore, since $c_{0}, c, c_{1}, \ldots, c_{m}$ are consecutive blocks of integers, $\operatorname{cr}(\gamma)=\operatorname{cr}\left(\gamma_{0}\right)+\operatorname{cr}\left(\gamma^{\prime}\right)$. Therefore $q^{\operatorname{cr}(\gamma)} M_{m+2}(\gamma)=q^{\operatorname{cr}\left(\gamma_{0}\right)} M_{2}\left(\gamma_{0}\right) q^{\operatorname{cr}\left(\gamma^{\prime}\right)} M_{m}\left(\gamma^{\prime}\right)$ and Lemmas 4.4 and 4.5 together imply that the first sum simplifies,

$$
\begin{array}{r}
\tau\left(X_{N, v_{0}} X_{N, u} \prod_{j=1}^{m} X_{N, v_{j}}^{\eta_{j}} Y_{N, v_{j}}^{1-\eta_{j}}\right)=\tau\left(X_{N, v_{0}} X_{N, u}\right) \tau\left(\prod_{j=1}^{m} X_{N, v_{j}}^{\eta_{j}} Y_{N, v_{j}}^{1-\eta_{j}}\right) \\
+\sum_{j=1}^{m} \sum_{\gamma \in \mathcal{H}_{j}\left(t_{\eta}\right)} q^{\operatorname{cr}(\gamma)} M_{m+2}(\gamma)+O(1 / N)
\end{array}
$$

For $i \in\{1, \ldots, n\}$, let

$$
\tilde{t}_{\eta}(i)= \begin{cases}-t_{\eta}(i) & \text { if } i \in c \\ t_{\eta}(i) & \text { otherwise }\end{cases}
$$

Since $Y_{N, u}$ differs from $X_{N, u}$ only in the sign of $\left.t\right|_{c}$, and since $\mathcal{H}_{0}\left(\tilde{t}_{\eta}\right)=\emptyset$, the same reasoning as above gives

$$
\tau\left(X_{N, v_{0}} Y_{N, u} \prod_{j=1}^{m} X_{N, v_{j}}^{\eta_{j}} Y_{N, v_{j}}^{1-\eta_{j}}\right)=\sum_{j=1}^{m} \sum_{\gamma \in \mathcal{H}_{j}\left(\tilde{t}_{\eta}\right)} q^{\mathrm{cr}(\gamma)} M_{m+2}(\gamma)+O(1 / N) .
$$

In view of (4.17) and (4.18), to prove (4.14), it is enough to show that for every $j \geq 1$,

$$
\sum_{\eta} \sum_{\gamma \in \mathcal{H}_{j}\left(t_{\eta}\right)} q^{\mathrm{cr}(\gamma)} M_{m+2}(\gamma)=\sum_{\eta} \sum_{\gamma \in \mathcal{H}_{j}\left(\tilde{t}_{\eta}\right)} q^{\operatorname{cr}(\gamma)} M_{m+2}(\gamma) .
$$


If $\gamma \in \mathcal{H}_{j}\left(t_{\eta}\right)$ then (4.13) gives

$$
M_{m+2}(\gamma)= \begin{cases}0 & \text { if } \eta_{j}=0 \\ M_{2}\left(\gamma_{j}\right) M_{m}\left(\gamma_{j}^{\prime}\right) & \text { if } \eta_{j}=1\end{cases}
$$

where $\gamma_{j}$ is the pairing on $c \cup c_{j}$ and $\gamma_{j}^{\prime}$ is the remaining set of pairs. On the other hand, if $\gamma \in \mathcal{H}_{j}\left(\tilde{t}_{\eta}\right)$ then

$$
M_{m+2}(\gamma)= \begin{cases}0 & \text { if } \eta_{j}=1 \\ M_{2}\left(\gamma_{j}\right) M_{m}\left(\gamma_{j}^{\prime}\right) & \text { if } \eta_{j}=0\end{cases}
$$

as the joint moments of $X_{N, u}, X_{N, c_{j}}$ are the same as the moments of $Y_{N, u}, Y_{N, c_{j}}$ and $t=\tilde{t}$ outside of $c \cup c_{j}$. The later also implies that with $\eta^{\prime}=\eta$ except for one entry $\eta^{\prime}(j)=1-\eta(j)$, we have

$$
\mathcal{H}_{j}\left(t_{\eta}\right)=\mathcal{H}_{j}\left(\tilde{t}_{\eta^{\prime}}\right)
$$

Thus summing over $\eta^{\prime}$ on the left hand side of (4.19) we get the right hand side, ending the proof.

Proof of Theorem 1.7. If $\lambda=0$ then all moments of $Z_{M, N}$ converge to zero, which we will consider as a normal or a semicircle law of variance 0 . Through the remainder of the proof we assume that $\lambda>0$. Since

$$
\left(Q\left(\mathbf{W}_{1}, \ldots, \mathbf{W}_{s}\right)-\tau\left(Q\left(\mathbf{W}_{1}, \ldots, \mathbf{W}_{s}\right)\right)\right)^{m}
$$

can be written as a linear combination of centered monomials of the form that appears on the left hand side of (4.6), combining Lemmas 4.4 and 4.5. we see that all moments converge. Furthermore, from (2.14) we see that the $n$-th moment grows at the rate bounded by a multiple of $\# \mathcal{F}_{n}=1 \cdot 3 \cdot \ldots \cdot(2 n-1)$, so in the self-adjoint case the limiting law is uniquely determined by moments.

When $q=1$ and $Q$ is real symmetric, the two copies $X_{N}, Y_{N}$ of $Z_{M, N}$ are independent and converge jointly to a pair of independent identically distributed self-adjoint (i.e., real) random variables $X, Y$. From Theorem 4.1, we see that $\mathbb{E}\left((X-Y)^{2} p(X+Y)\right)=2 \mathbb{E}\left(X^{2}\right) \mathbb{E}(p(X+Y))$ for all polynomials $p$. By the previous estimate for the moments, the characteristic function of $X+Y$ is analytic at 0 , so the moment problem is unique and we can replace polynomial $p$ by any bounded measurable function. Thus $\operatorname{Var}(\mathrm{X} \mid \mathrm{X}+\mathrm{Y})=$ const, which is known to imply that $X$ is normal, see [26, Corollary 4.1].

When $q=0$ and $Q$ is real symmetric, the two copies $X_{N}, Y_{N}$ of $Z_{M, N}$ are free and converge jointly to a pair of free identically distributed self-adjoint noncommutative random variables $X, Y$. From Theorem 4.1, we see that $\tau\left((X-Y)^{2} p(X+Y)\right)=2 \tau\left(X^{2}\right) \tau(p(X+Y))$ for all polynomials $p$. For $q=0$, the moments of $X$ are bounded by the number of non-crossing elements in $\mathcal{F}_{n}$; this is the $2 n$-th Catalan number, so the support of $X+Y$ is bounded and we deduce that $\operatorname{Var}(\mathrm{X} \mid \mathrm{X}+\mathrm{Y})=$ const, which is known to imply that $X$ has semicircle law, see [5, Theorem 3.2] for a more general result.

\subsection{Concluding examples}

The following computer-assisted calculations give low order moments of some polynomials in $q$-Wishart matrices. Together, they illustrate that the limit law depends on the polynomial in a nontrivial way.

Example 4.6. In the simplest case $\mathbf{W}_{N} \in \mathcal{W}_{q}\left(\frac{1}{N} I_{N}, I_{M}\right)$ and $M=\lambda N$, we have $\tau\left(\operatorname{tr}\left(\mathbf{W}_{N}\right)\right)=\lambda N$. The first six moments for the limit of $X_{N}=\operatorname{tr}\left(\mathbf{W}_{N}\right)-N \lambda$ are consistent with the $q^{4}$-Gaussian law: with $s^{2}:=\tau\left(X_{N}^{2}\right)=$ $(1+q) \lambda$, we have $\tau\left(X_{N}^{3}\right) \rightarrow 0$,

$$
\tau\left(X_{N}^{4}\right) / s^{4} \rightarrow\left(2+q^{4}\right), \tau\left(X_{N}^{5}\right) \rightarrow 0 ; \tau\left(X_{N}^{6}\right) / s^{6} \rightarrow 5+6 q^{4}+3 q^{8}+q^{12} .
$$

Example 4.7. To see whether one could identify the limit law for a second-order polynomial in $\mathbf{W} \in$ $\mathcal{W}_{q}\left(\frac{1}{N} I_{N}, I_{M}\right)$, we computed the first moments of the limit as $N \rightarrow \infty$ and $M / N \rightarrow \lambda$ of

$$
X_{N}=\operatorname{tr}\left(\mathbf{W}^{2}\right)-a \operatorname{tr}(\mathbf{W})-\tau\left(\operatorname{tr}\left(\mathbf{W}^{2}\right)-a \operatorname{tr}(\mathbf{W})\right)
$$

The general expression for the asymptotic variance is cumbersome, but it simplifies to $\tau\left(X_{N}^{2}\right) \rightarrow \lambda^{2}\left(q^{6}+q^{4}+\right.$ $\left.q^{2}+1\right)$ when $a=1+q^{2}+2 \lambda$. For this value of a, the normalized fourth moments $\tau\left(X_{N}^{4}\right) /\left(\tau\left(X_{N}^{2}\right)\right)^{2}$ converge to $2+q^{16}$, which is the fourth moment of the $q^{16}$-Gaussian law of unit variance. 
Example 4.8. Suppose $\mathbf{W}_{1}, \mathbf{W}_{2} \in \mathcal{W}_{q}\left(\frac{1}{N} I_{N}, I_{M}\right)$ are q-orthogonal. Then $\tau\left(\operatorname{tr}\left(\mathbf{W}_{1} \mathbf{W}_{2}\right)\right)=M^{2} / N$, see Example 2.10, and from Table 1 we see that $\operatorname{Var}\left(\operatorname{tr}\left(\mathbf{W}_{1} \mathbf{W}_{2}\right)\right)=\left(1+q^{2}\right) q^{4} M^{2} / N^{2}+2(1+q) M^{3} / N^{3}+(1+q) q^{4} / N^{3}$. Denote $X_{N}=\operatorname{tr}\left(\mathbf{W}_{1} \mathbf{W}_{2}\right)-N \lambda^{2}$, and suppose $M=M(N)=\lambda N+O(1 / N)$. Then $\lim _{N \rightarrow \infty} \tau\left(X_{N}^{2}\right)=$ $\lambda^{2}\left(q^{4}+q^{6}+2 \lambda+2 q \lambda\right)$, and computer-assisted calculations give $\lim _{N \rightarrow \infty} \tau\left(X_{N}^{3}\right)=0$ and

$$
\lim _{N \rightarrow \infty} \tau\left(X_{N}^{4}\right)=\lambda^{4}\left(q^{8}\left(1+q^{2}\right)^{2}\left(2+q^{16}\right)+4 q^{4}(1+q)\left(1+q^{2}\right)\left(2+q^{8}\right) \lambda+4(1+q)^{2}\left(2+q^{4}\right) \lambda^{2}\right) .
$$

After normalizing by the variance, we see that in this case the fourth moments converge to a rational function of $q$.

Funding

Funding for this work was provided by the National Science Foundation (\#DMS-0504198).

Acknowledgement

We would like to thank J. Mingo and R. Speicher for helpful comments on the earlier draft of this paper and for information about their research [32] and [31, to P. Śniady for clarifying certain aspects of the Brauer product, and to V. Pierce for a discussion that motivated this research and for further help with computer-assisted computations. We further thank an anonymous referee for a simpler proof of Lemma 2.20 and for comments that improved presentation.

\section{References}

[1] Anderson, T. W. An introduction to multivariate statistical analysis, third ed. Wiley Series in Probability and Statistics. Wiley-Interscience [John Wiley \& Sons], Hoboken, NJ, 2003.

[2] Arharov, L. V. Limit theorems for the characteristic roots of a sample covariance matrix. Dokl. Akad. Nauk SSSR 199 (1971), 994-997.

[3] Biane, P. Some properties of crossings and partitions. Discrete Math. 175, 1-3 (1997), 41-53.

[4] Billingsley, P. Probability and measure, third ed. Wiley Series in Probability and Mathematical Statistics. John Wiley \& Sons Inc., New York, 1995. A Wiley-Interscience Publication.

[5] BożEJKo, M., And Bryc, W. On a class of free Lévy laws related to a regression problem. J. Funct. Anal. 236 (2006), 59-77. arxiv.org/abs/math.OA/0410601.

[6] Bożejko, M., Kümmerer, B., And Speicher, R. q-Gaussian processes: non-commutative and classical aspects. Comm. Math. Phys. 185, 1 (1997), 129-154.

[7] Bożejko, M., And Speicher, R. An example of a generalized Brownian motion. Comm. Math. Phys. 137, 3 (1991), 519-531.

[8] Brauer, R. On algebras which are connected with the semisimple continuous groups. The Annals of Mathematics 38, 4 (oct 1937), 857-872.

[9] BRYC, W. Asymptotic normality for traces of polynomials in independent complex Wishart matrices. Probab. Theor. Rel. Fields 140 (2008), 383-405. arxiv.org/abs/math.PR/0701318.

[10] Burda, Z., Jarosz, A., Jurkiewicz, J., Nowak, M. A., Papp, G., and Zahed, I. Applying free random variables to random matrix analysis of financial data. arXiv.org:physics/0603024, 2006.

[11] Chuah, C.-N., Tse, D. N. C., Kahn, J. M., and Valenzuela, R. A. Capacity scaling in Mimo wireless systems under correlated fading. IEEE Trans. Inform. Theory 48, 3 (2002), 637-650.

[12] Collins, B., Mingo, J. A., Sniady, P., and Speicher, R. Second Order Freeness and Fluctuations of Random Matrices, III. Higher order freeness and free cumulants. Doc. Math. 12 (2007), 1-70. http://arxiv.org.math.OA/0606431.

[13] Collins, B., ANd Śniady, P. Integration with respect to the Haar measure on unitary, orthogonal and symplectic group. Comm. Math. Phys. 264, 3 (2006), 773-795.

[14] Frisch, U., And Bourret, R. Parastochastics. J. Math. Phys. 11, 2 (1970), 364-390. 
[15] Goulden, I. P., And Jackson, D. M. Connection coefficients, matchings, maps and combinatorial conjectures for Jack symmetric functions. Trans. Amer. Math. Soc. 348, 3 (1996), 873-892.

[16] Graczyk, P., Letac, G., and Massam, H. The complex Wishart distribution and the symmetric group. Ann. Statist. 31, 1 (2003), 287-309.

[17] Graczyk, P., Letac, G., And Massam, H. The hyperoctahedral group, symmetric group representations and the moments of the real Wishart distribution. J. Theoret. Probab. 18, 1 (2005), 1-42.

[18] Graczyk, P., And Vostrikova, L. The moments of Wishart processes via Itô calculus. Teor. Veroyatn. Primen. 51, 4 (2006), 732-751.

[19] Guionnet, A., Jones, V. F. R., and Shlyakhtenko, D. Random matrices, free probability, planar algebras and subfactors, 2007. arXiv.org:0712.2904.

[20] Hanlon, P. J., Stanley, R. P., And Stembridge, J. R. Some combinatorial aspects of the spectra of normally distributed random matrices. In Hypergeometric functions on domains of positivity, Jack polynomials, and applications (Tampa, FL, 1991), vol. 138 of Contemp. Math. Amer. Math. Soc., Providence, RI, 1992, pp. 151-174.

[21] Hiai, F., AND Petz, D. The semicircle law, free random variables and entropy, vol. 77 of Mathematical Surveys and Monographs. American Mathematical Society, Providence, RI, 2000.

[22] Jacques, A. Sur le genre d'une paire de substitutions. C. R. Acad. Sci. Paris Sér. A-B 267 (1968), A625-A627.

[23] Jonsson, D. Some limit theorems for the eigenvalues of a sample covariance matrix. J. Multivariate Anal. 12, 1 (1982), 1-38.

[24] Kuperberg, G. A tracial quantum central limit theorem. Trans. Amer. Math. Soc. 357, 2 (2005), 459-471 (electronic).

[25] Kusalik, T., Mingo, J. A., And Speicher, R. Orthogonal polynomials and fluctuations of random matrices. J. Reine Angew. Math. 604 (2007), 1-46. arXiv:math.OA/0503169.

[26] LAнA, R. G. On a characterization of the normal distribution from properties of suitable linear statistics. Ann. Math. Statist. 28 (1957), 126-139.

[27] Lando, S. K., And Zvonkin, A. K. Graphs on surfaces and their applications, vol. 141 of Encyclopaedia of Mathematical Sciences. Springer-Verlag, Berlin, 2004. With an appendix by Don B. Zagier, LowDimensional Topology, II.

[28] Lu, I.-L., And Richards, D. S. P. MacMahon's master theorem, representation theory, and moments of Wishart distributions. Adv. in Appl. Math. 27, 2-3 (2001), 531-547. Special issue in honor of Dominique Foata's 65th birthday (Philadelphia, PA, 2000).

[29] Machì, A. The Riemann-Hurwitz formula for the centralizer of a pair of permutations. Arch. Math. (Basel) 42, 3 (1984), 280-288.

[30] Mingo, J., And NicA, A. Random unitaries in non-commutative tori, and an asymptotic model for q-circular systems. Indiana Univ. Math. J. 50, 2 (2001), 953-987.

[31] Mingo, J., NicA, A., And Speicher, R. Random matrices with non-commutative Gaussian entries: Fluctuations around the semicircular limit. (private communication), 2007.

[32] Mingo, J. A. Personal communication, 2008.

[33] Mingo, J. A., And NicA, A. Annular noncrossing permutations and partitions, and second-order asymptotics for random matrices. Int. Math. Res. Not. 28 (2004), 1413-1460.

[34] Mingo, J. A., Speicher, R., And TAn, E. Second order cumulants of products, 2007. arXiv.org:0708.0586.

[35] Muirhead, R. J. Aspects of multivariate statistical theory. John Wiley \& Sons Inc., New York, 1982. Wiley Series in Probability and Mathematical Statistics. 
[36] Nica, A., Shlyakhtenko, D., And Speicher, R. R-cyclic families of matrices in free probability. $J$. Funct. Anal. 188, 1 (2002), 227-271. arXiv:math.OA/0101025.

[37] NicA, A., And Speicher, R. On the multiplication of free $N$-tuples of noncommutative random variables. Amer. J. Math. 118, 4 (1996), 799-837.

[38] PARIsi, G. D-dimensional arrays of Josephson junctions, spin glasses and $q$-deformed harmonic oscillators. J. Phys. A 27, 23 (1994), 7555-7568.

[39] RyAn, Ø. On the limit distributions of random matrices with independent or free entries. Comm. Math. Phys. 193, 3 (1998), 595-626.

[40] Shlyakhtenko, D. Limit distributions of matrices with bosonic and fermionic entries. In Free probability theory (Waterloo, ON, 1995), vol. 12 of Fields Inst. Commun. Amer. Math. Soc., Providence, RI, 1997, pp. 241-252.

[41] Speicher, R. Combinatorial theory of the free product with amalgamation and operator-valued free probability theory. Mem. Amer. Math. Soc. 132, 627 (1998), x+88.

[42] Stanley, R. P. Enumerative combinatorics. Vol. 2, vol. 62 of Cambridge Studies in Advanced Mathematics. Cambridge University Press, Cambridge, 1999. With a foreword by Gian-Carlo Rota and appendix 1 by Sergey Fomin.

[43] Thorbjørnsen, S. Mixed moments of Voiculescu's Gaussian random matrices. J. Funct. Anal. 176,2 (2000), 213-246.

[44] van Leeuwen, H., And MaAssen, H. The $q$-harmonic oscillator in a lattice model. J. Math. Phys. 39, 12 (1998), 6424-6440.

[45] Voiculescu, D. V., Dykema, K. J., And NicA, A. Free random variables. American Mathematical Society, Providence, RI, 1992.

[46] Wishart, J. The generalised product moment distribution in samples from a normal multivariate population. Biometrika 20A, 1/2 (jul 1928), 32-52. 